\title{
Application of Biologically Based Lumping to Investigate the Toxicokinetic Interactions of a Complex Gasoline Mixture
}

\section{Supplemental Material}

Micah N. Jasper, Sheppard A. Martin, Wendy M. Oshiro, Jermaine Ford, Philip J. Bushnell, and Hisham El-Masri*

National Health and Environmental Effects Research Laboratory, Office of Research and Development, U. S. Environmental Protection Agency, Research Triangle Park, North Carolina

Key words: PBPK, mixtures, interaction, competitive inhibition, chemical lumping, clustering, BBLM

*Contact author: Hisham El-Masri, US EPA/NHEERL/ISTD/SSD.

Email: el-masri.hisham@epa.gov

This manuscript has been reviewed in accordance with the policy of the National Health and Environmental Effects Research Laboratory, U.S. Environmental Protection Agency, and approved for publication. Approval does not signify that the contents necessarily reflect the views and policies of the Agency, nor does mention of trade names or commercial products constitute endorsement or recommendation for use.

\section{Supplemental Material Summary}

Pages: 25

Tables: 8

Figures: 5

Equations: 11 


\section{Supplemental Table 1}

Chemical Specific Parameters for the 10 Target Chemicals

\begin{tabular}{|c|c|c|c|c|c|c|c|c|c|c|c|c|c|c|}
\hline No. & Name & Percent & ppmv & MW & BP & PBA & PFat & PBrn & PLiv & PMsl & PKid & PLng & $V \max C$ & KmL \\
\hline 1 & n-Pentane & 9.339 & 837.5 & 72.15 & 36.06 & 0.38 & 104.21 & 5.79 & 5.53 & 1.84 & 1.58 & $\underline{0.05}$ & 0.29 & 0.40 \\
\hline 2 & 2-Methylpentane & 3.794 & 283.5 & 86.18 & 60.21 & 0.41 & 212.20 & 9.27 & 10.98 & 7.07 & 4.88 & $\underline{0.20}$ & 0.28 & 1.02 \\
\hline 3 & Toluene & 2.178 & 139.5 & 92.14 & 110.6 & 15.90 & 62.26 & 2.29 & 5.26 & 1.74 & 1.12 & $\underline{0.79}$ & 3.44 & 0.13 \\
\hline 4 & n-Hexane & 1.796 & 134.2 & 86.18 & 68.73 & 1.72 & 92.44 & 2.91 & 3.02 & 1.69 & 1.74 & $\underline{0.14}$ & 1.27 & 3.65 \\
\hline 5 & Cyclohexane & 0.925 & 69.1 & 84.16 & 80.7 & 1.39 & 169.06 & 7.70 & 5.67 & 0.74 & 5.18 & $\underline{0.21}$ & 3.79 & 5.81 \\
\hline 6 & Benzene & 0.641 & 47.9 & 78.11 & 80.08 & 14.10 & 30.67 & 1.28 & 1.21 & 0.73 & 0.86 & $\underline{0.73}$ & 2.11 & 0.10 \\
\hline 7 & m-Xylene & 0.198 & 11.1 & 106.17 & 139.07 & 46.00 & 45.48 & 0.84 & 1.98 & 0.91 & 0.65 & $\underline{0.56}$ & 6.49 & 0.45 \\
\hline 8 & Ethylbenzene & 0.103 & 5.8 & 106.17 & 136.1 & 30.45 & 51.10 & 1.16 & 1.98 & 0.85 & 0.90 & $\underline{0.74}$ & 4.58 & 0.52 \\
\hline 9 & p-Xylene & 0.100 & 5.6 & 106.17 & 138.23 & 41.30 & 42.32 & 0.91 & 2.18 & 0.93 & 0.71 & $\underline{0.57}$ & 5.31 & 0.72 \\
\hline 10 & o-Xylene & 0.092 & 5.2 & 106.17 & 144.5 & 44.30 & 54.27 & 1.03 & 2.44 & 1.16 & 0.80 & $\underline{0.70}$ & 5.31 & 0.72 \\
\hline
\end{tabular}

Values in bold are experimental rat data from Meulenberg et al. ${ }^{1}$ and Price and Krishnan ${ }^{2}$. Values in bold and italics are experimental human data from Meulenberg et al. ${ }^{1}$. Values in italics are estimated data using the BP method in Perbellini, et al. ${ }^{3}$ for PC values and the QSPR method in Price and Krishnan ${ }^{2}$ for the Vmax and Km values. The underlined PLng values were optimized to fit experimental exposure data in this research. 
Supplemental Table 2

Chemical Data for Partition Coefficient (PC) Estimation for Select ${ }^{*}$ Muelenberg Chemicals

\begin{tabular}{|c|c|c|c|c|c|c|c|c|c|c|c|c|c|c|c|c|c|}
\hline No. & Compound Name & Alternate Names & BP & VP & $\log$ Kow & MW & $\mathrm{CH}_{3}$ & $\mathrm{CH}_{2}$ & $\mathbf{C H}$ & $\mathbf{C}$ & $\mathrm{C}=\mathrm{C}$ & $\mathbf{H}$ & $\mathbf{B r}$ & $\mathbf{C l}$ & $\mathbf{F}$ & $\mathbf{A C}$ & H_AC \\
\hline 1 & Ethane & & -88.6 & 31500 & 1.81 & 30.06904 & 2 & 0 & 0 & 0 & 0 & 0 & 0 & 0 & 0 & 0 & 0 \\
\hline 2 & n-Butane & & -0.5 & 1820 & 2.89 & 58.1222 & 2 & 2 & 0 & 0 & 0 & 0 & 0 & 0 & 0 & 0 & 0 \\
\hline 3 & n-Pentane & & 36 & 514 & 3.39 & 72.14878 & 2 & 3 & 0 & 0 & 0 & 0 & 0 & 0 & 0 & 0 & 0 \\
\hline 4 & n-Hexane & & 68.7 & 151 & 3.9 & 86.17536 & 2 & 4 & 0 & 0 & 0 & 0 & 0 & 0 & 0 & 0 & 0 \\
\hline 5 & n-Heptane & & 98.5 & 46 & 4.66 & 100.2019 & 2 & 5 & 0 & 0 & 0 & 0 & 0 & 0 & 0 & 0 & 0 \\
\hline 6 & n-Octane & & 125.6 & 14.1 & 5.18 & 114.2285 & 2 & 6 & 0 & 0 & 0 & 0 & 0 & 0 & 0 & 0 & 0 \\
\hline 7 & n-Decane & & 174.1 & 1.43 & 5.01 & 142.2817 & 2 & 8 & 0 & 0 & 0 & 0 & 0 & 0 & 0 & 0 & 0 \\
\hline 8 & Ethylene & & -103.7 & 52100 & 1.13 & 28.05316 & 0 & 0 & 0 & 0 & 1 & 4 & 0 & 0 & 0 & 0 & 0 \\
\hline 9 & 2,2-Dimethylbutane & & 49.7 & 319 & 3.82 & 86.17536 & 4 & 1 & 0 & 1 & 0 & 0 & 0 & 0 & 0 & 0 & 0 \\
\hline 10 & 2-Methyl-1,3-butadiene & Isoprene & 34 & 550 & 2.42 & 68.11702 & 1 & 0 & 0 & 0 & 2 & 6 & 0 & 0 & 0 & 0 & 0 \\
\hline 11 & 2-Methylpentane & Isohexane & 60.2 & 211 & 3.21 & 86.17536 & 3 & 2 & 1 & 0 & 0 & 0 & 0 & 0 & 0 & 0 & 0 \\
\hline 12 & 3-Methylpentane & & 63.2 & 190 & 3.6 & 86.17536 & 3 & 2 & 1 & 0 & 0 & 0 & 0 & 0 & 0 & 0 & 0 \\
\hline 13 & 3-Methylhexane & & 90 & 66 & 3.71 & 100.2019 & 3 & 3 & 1 & 0 & 0 & 0 & 0 & 0 & 0 & 0 & 0 \\
\hline 14 & 2,3,4-Trimethylpentane & & 79.2 & 105 & 3.67 & 100.2019 & 5 & 0 & 3 & 0 & 0 & 0 & 0 & 0 & 0 & 0 & 0 \\
\hline 15 & 2,2,4-Trimethylpentane & & 99.2 & 49.3 & 4.09 & 114.2285 & 5 & 1 & 1 & 1 & 0 & 0 & 0 & 0 & 0 & 0 & 0 \\
\hline 16 & Cyclopropane & & -32.8 & 5410 & 1.72 & 42.07974 & 0 & 3 & 0 & 0 & 0 & 0 & 0 & 0 & 0 & 0 & 0 \\
\hline 17 & Cyclopentane & & 49.3 & 318 & 3 & 70.1329 & 0 & 3 & 0 & 0 & 1 & 2 & 0 & 0 & 0 & 0 & 0 \\
\hline 18 & Cyclohexane & & 80.7 & 96.9 & 3.44 & 84.15948 & 0 & 6 & 0 & 0 & 0 & 0 & 0 & 0 & 0 & 0 & 0 \\
\hline 19 & Cycloheptane & & 118.4 & 21.6 & 4 & 98.18606 & 0 & 7 & 0 & 0 & 0 & 0 & 0 & 0 & 0 & 0 & 0 \\
\hline 20 & Methylcyclopentane & & 71.8 & 138 & 3.37 & 84.15948 & 1 & 4 & 1 & 0 & 0 & 0 & 0 & 0 & 0 & 0 & 0 \\
\hline 21 & Benzene & & 80 & 94.8 & 2.13 & 78.11184 & 0 & 0 & 0 & 0 & 0 & 0 & 0 & 0 & 0 & 1 & 6 \\
\hline 22 & Toluene & methylbenzene & 110.6 & 28.4 & 2.73 & 92.13842 & 1 & 0 & 0 & 0 & 0 & 0 & 0 & 0 & 0 & 1 & 5 \\
\hline 23 & o-Xylene & 1,2-dimethylbenzene & 138.5 & 7.99 & 3.12 & 106.165 & 2 & 0 & 0 & 0 & 0 & 0 & 0 & 0 & 0 & 1 & 4 \\
\hline 24 & m-Xylene & 1,3-dimethylbenzene & 139.1 & 8.29 & 3.2 & 106.165 & 2 & 0 & 0 & 0 & 0 & 0 & 0 & 0 & 0 & 1 & 4 \\
\hline 25 & p-Xylene & 1,4-dimethylbenzene & 138.3 & 8.84 & 3.15 & 106.165 & 2 & 0 & 0 & 0 & 0 & 0 & 0 & 0 & 0 & 1 & 4 \\
\hline
\end{tabular}




\begin{tabular}{|c|c|c|c|c|c|c|c|c|c|c|c|c|c|c|c|c|c|}
\hline 26 & Ethylbenzene & & 136.1 & 9.6 & 3.15 & 106.165 & 1 & 1 & 0 & 0 & 0 & 0 & 0 & 0 & 0 & 1 & 5 \\
\hline 27 & Styrene & $\begin{array}{l}\text { Vinylbenzene, } \\
\text { Phenylethylene }\end{array}$ & 145 & 6.4 & 2.95 & 104.1491 & 0 & 0 & 0 & 0 & 1 & 3 & 0 & 0 & 0 & 1 & 5 \\
\hline 28 & 1,3,5-Trimethylbenzene & mesitylene & 164.7 & 2.1 & 3.42 & 120.1916 & 3 & 0 & 0 & 0 & 0 & 0 & 0 & 0 & 0 & 1 & 3 \\
\hline 29 & $1,2,4$-Trimethylbenzene & pseudocumene & 169.3 & 2.1 & 3.63 & 120.1916 & 3 & 0 & 0 & 0 & 0 & 0 & 0 & 0 & 0 & 1 & 3 \\
\hline 30 & 1,2,3-Trimethylbenzene & hemimellitene & 176.1 & 1.69 & 3.66 & 120.1916 & 3 & 0 & 0 & 0 & 0 & 0 & 0 & 0 & 0 & 1 & 3 \\
\hline 31 & Propylbenzene & & 159.2 & 3.42 & 3.69 & 120.1916 & 1 & 2 & 0 & 0 & 0 & 0 & 0 & 0 & 0 & 1 & 5 \\
\hline 32 & Allylbenzene & & 156 & 3.03 & 3.23 & 118.1757 & 0 & 1 & 0 & 0 & 1 & 3 & 0 & 0 & 0 & 1 & 5 \\
\hline 33 & Cumene & Isopropylbenzene & 152.4 & 4.5 & 3.66 & 120.1916 & 2 & 0 & 1 & 0 & 0 & 0 & 0 & 0 & 0 & 1 & 5 \\
\hline 34 & m-Methylstyrene & $\begin{array}{l}\text { 1-vinyl-3-methylbenzene, } \\
\text { 3-Vinyltoluene }\end{array}$ & 164 & 1.7 & 3.44 & 118.1757 & 1 & 0 & 0 & 0 & 1 & 3 & 0 & 0 & 0 & 1 & 4 \\
\hline 35 & p-Methylsytrene & $\begin{array}{l}\text { 1-vinyl-4-methylbenzene, } \\
\text { 4-Vinyltoluene }\end{array}$ & 172.8 & 1.81 & 3.44 & 118.1757 & 1 & 0 & 0 & 0 & 1 & 3 & 0 & 0 & 0 & 1 & 4 \\
\hline 36 & Chloromethane & Methylchloride & -24 & 4300 & 0.91 & 50.48752 & 1 & 0 & 0 & 0 & 0 & 0 & 0 & 1 & 0 & 0 & 0 \\
\hline 37 & Dichloromethane & & 40 & 435 & 1.25 & 84.93258 & 0 & 1 & 0 & 0 & 0 & 0 & 0 & 2 & 0 & 0 & 0 \\
\hline 38 & Chloroform & trichloromethane & 61.1 & 197 & 1.97 & 119.3776 & 0 & 0 & 1 & 0 & 0 & 0 & 0 & 3 & 0 & 0 & 0 \\
\hline 39 & Carbon-tetrachloride & $\begin{array}{l}\text { Tetrachloromethane, } \\
\text { Perchloromethane }\end{array}$ & 76.8 & 115 & 2.83 & 153.8227 & 0 & 0 & 0 & 1 & 0 & 0 & 0 & 4 & 0 & 0 & 0 \\
\hline 40 & Chloroethane & monochloroethane & 12.3 & 1010 & 1.43 & 64.5141 & 1 & 0 & 1 & 0 & 0 & 0 & 0 & 1 & 0 & 0 & 0 \\
\hline 41 & 1,1-Dichloroethane & & 57.4 & 227 & 1.79 & 98.95916 & 1 & 0 & 1 & 0 & 0 & 0 & 0 & 2 & 0 & 0 & 0 \\
\hline 42 & 1,2-Dichloroethane & & 83.5 & 78.9 & 1.48 & 98.95916 & 0 & 2 & 0 & 0 & 0 & 0 & 0 & 2 & 0 & 0 & 0 \\
\hline 43 & 1,1,1-Trichloroethane & & 74 & 124 & 2.49 & 133.4042 & 1 & 0 & 1 & 0 & 0 & 0 & 0 & 3 & 0 & 0 & 0 \\
\hline 44 & 1,1,2-Tetrachloroethane & & 113.8 & 23 & 1.89 & 133.4042 & 0 & 1 & 1 & 0 & 0 & 0 & 0 & 3 & 0 & 0 & 0 \\
\hline 45 & 1,1,1,2-Tetrachloroethane & & 130.5 & 12 & 2.93 & 167.8493 & 0 & 1 & 0 & 1 & 0 & 0 & 0 & 4 & 0 & 0 & 0 \\
\hline 46 & 1,1,2,2-Tetrachloroethane & & 146.5 & 13.3 & 2.39 & 169.8616 & 0 & 0 & 2 & 0 & 0 & 0 & 0 & 4 & 0 & 0 & 0 \\
\hline 47 & Pentachloroethane & & 159.8 & 3.5 & 3.22 & 202.2943 & 0 & 0 & 1 & 1 & 0 & 0 & 0 & 5 & 0 & 0 & 0 \\
\hline 48 & Hexachloroethane & Perchloroethane & 154.45 & 0.21 & 4.14 & 236.7394 & 0 & 0 & 2 & 0 & 0 & 0 & 0 & 6 & 0 & 0 & 0 \\
\hline 49 & Chloroethylene & vinylchloride & -13.3 & 2980 & 1.62 & 62.49822 & 0 & 0 & 0 & 0 & 1 & 3 & 0 & 1 & 0 & 0 & 0 \\
\hline 50 & 1,1-Dichloroethylene & $\begin{array}{l}\text { Vinylidene-Chloride, 1,1- } \\
\text { Dichloroethene }\end{array}$ & 31.6 & 634 & 2.13 & 96.94328 & 0 & 0 & 0 & 0 & 1 & 2 & 0 & 2 & 0 & 0 & 0 \\
\hline 51 & cis-1,2-Dichloroethylene & (Z)-1,2-Dichloroethene & 55 & 201 & 1.86 & 96.943 & 0 & 0 & 0 & 0 & 1 & 2 & 0 & 2 & 0 & 0 & 0 \\
\hline 52 & trans-1,2-Dichloroethylene & (E)-1,2-Dichloroethene & 55 & 201 & 2.09 & 96.943 & 0 & 0 & 0 & 0 & 1 & 2 & 0 & 2 & 0 & 0 & 0 \\
\hline 53 & Trichloroethylene & & 87.2 & 69 & 2.42 & 131.3883 & 0 & 0 & 0 & 0 & 1 & 1 & 0 & 3 & 0 & 0 & 0 \\
\hline
\end{tabular}




\begin{tabular}{|c|c|c|c|c|c|c|c|c|c|c|c|c|c|c|c|c|c|}
\hline 54 & Tetrachloroethylene & perchloroethylene & 121.3 & 18.5 & 3.4 & 165.8334 & 0 & 0 & 0 & 0 & 1 & 0 & 0 & 4 & 0 & 0 & 0 \\
\hline 55 & 1-Chloropropane & & 46.5 & 345 & 2.04 & 78.54068 & 1 & 2 & 0 & 0 & 0 & 0 & 0 & 1 & 0 & 0 & 0 \\
\hline 56 & 2-Chloropropane & & 35.7 & 515 & 1.9 & 78.54068 & 2 & 0 & 1 & 0 & 0 & 0 & 0 & 1 & 0 & 0 & 0 \\
\hline 57 & 1,2-Dichloropropane & $\begin{array}{l}\text { 1,1-Dichloropropane, } \\
\text { Dichloropropane }\end{array}$ & 95.5 & 53.3 & 1.98 & 112.9857 & 1 & 1 & 1 & 0 & 0 & 0 & 0 & 2 & 0 & 0 & 0 \\
\hline 58 & 3-Chloro-1-propylene & Allylchloride & 45.1 & 368 & 1.93 & 76.5248 & 0 & 1 & 0 & 0 & 1 & 3 & 0 & 1 & 0 & 0 & 0 \\
\hline 59 & 1-Chlorobutane & & 78.6 & 101 & 2.64 & 92.56726 & 1 & 3 & 0 & 0 & 0 & 0 & 0 & 1 & 0 & 0 & 0 \\
\hline 60 & 1-Chloropentane & & 107.8 & 32.9 & 2.73 & 106.5938 & 1 & 4 & 0 & 0 & 0 & 0 & 0 & 1 & 0 & 0 & 0 \\
\hline 61 & Chlorobenzene & & 131.7 & 12 & 2.84 & 112.5569 & 0 & 0 & 0 & 0 & 0 & 0 & 0 & 1 & 0 & 1 & 5 \\
\hline 62 & o-Dichlorobenzene & 1,2-Dichlorobenzene & 180 & 1.47 & 3.43 & 147.002 & 0 & 0 & 0 & 0 & 0 & 0 & 0 & 2 & 0 & 1 & 4 \\
\hline 63 & m-Dichlorobenzene & 1,3-Dichlorobenzene & 173 & 2.15 & 3.53 & 147.002 & 0 & 0 & 0 & 0 & 0 & 0 & 0 & 2 & 0 & 1 & 4 \\
\hline 64 & Dibromomethane & & 97 & 44.4 & 1.7 & 173.8346 & 0 & 1 & 0 & 0 & 0 & 0 & 2 & 0 & 0 & 0 & 0 \\
\hline 65 & 1,2-Dibromoethane & & 131.6 & 11.2 & 1.96 & 187.8612 & 0 & 2 & 0 & 0 & 0 & 0 & 2 & 0 & 0 & 0 & 0 \\
\hline 66 & Bromoethylene & Vinylbromide & 15.8 & 1030 & 1.57 & 106.9492 & 0 & 0 & 0 & 0 & 1 & 3 & 1 & 0 & 0 & 0 & 0 \\
\hline 67 & 1-Bromopropane & n-propylbromide & 71.1 & 111 & 2.1 & 122.9917 & 1 & 2 & 0 & 0 & 0 & 0 & 1 & 0 & 0 & 0 & 0 \\
\hline 68 & 2-Bromopropane & isopropylbromide & 59.5 & 216 & 2.14 & 122.9917 & 2 & 0 & 1 & 0 & 0 & 0 & 1 & 0 & 0 & 0 & 0 \\
\hline 71 & $\mathrm{CF}_{2} \mathrm{H}_{2}$ & Difluoromethane, R-32 & -51.6 & 12600 & 0.2 & 52.02339 & 0 & 1 & 0 & 0 & 0 & 0 & 0 & 0 & 2 & 0 & 0 \\
\hline 72 & $\mathrm{CF}_{4}$ & $\begin{array}{l}\text { Carbon-Tetrafluoride, } \\
\text { Perfluoromethane, } \\
\text { Tetrafluoromethane, R-14 }\end{array}$ & -128 & 175000 & 1.18 & 88.00431 & 0 & 0 & 0 & 1 & 0 & 0 & 0 & 0 & 4 & 0 & 0 \\
\hline 73 & $\mathrm{CF}_{3} \mathrm{CFH}_{2}$ & $\begin{array}{l}\text { 1,1,1,2-Tetrafluoroethane, } \\
\text { R-134a }\end{array}$ & -26 & 4990 & 1.68 & 102.0309 & 0 & 1 & 0 & 1 & 0 & 0 & 0 & 0 & 4 & 0 & 0 \\
\hline 74 & $\mathrm{CF}_{2} \mathrm{HCF}_{2} \mathrm{H}$ & $\begin{array}{l}\text { 1,1,2,2-Tetrafluoroethane, } \\
\text { R-134 }\end{array}$ & -19.9 & 3330 & 0.94 & 102.0309 & 0 & 0 & 2 & 0 & 0 & 0 & 0 & 0 & 4 & 0 & 0 \\
\hline 75 & $\mathrm{CF}_{2} \mathrm{HCH}_{3}$ & $\begin{array}{l}\text { 1,1-Difluoroethane, } \\
\text { F 152A }\end{array}$ & -24.9 & 4550 & 0.75 & 66.04997 & 1 & 0 & 1 & 0 & 0 & 0 & 0 & 0 & 2 & 0 & 0 \\
\hline 76 & $\mathrm{CF}_{2} \mathrm{HCF}_{2} \mathrm{CF}_{2} \mathrm{H}$ & 1,1,3,3-Tetrafluoropropane & -30.68 & 4550 & 1.43 & 116.058 & 0 & 1 & 2 & 0 & 0 & 0 & 0 & 0 & 4 & 0 & 0 \\
\hline 77 & $\mathrm{CFH}_{2} \mathrm{CH}_{2} \mathrm{CFH}_{2}$ & 1,3-Difluoropropane & 41.6 & 418 & 1.70 & 80.076 & 0 & 3 & 0 & 0 & 0 & 0 & 0 & 0 & 2 & 0 & 0 \\
\hline 78 & $\mathrm{CF}_{3} \mathrm{CF}_{2} \mathrm{CF}_{2} \mathrm{CF}_{2} \mathrm{H}$ & $\begin{array}{l}1,1,1,2,2,3,3,4,4- \\
\text { Nonafluorobutane }\end{array}$ & -10.69 & 2510 & 3.48 & 220.0364 & 0 & 0 & 1 & 3 & 0 & 0 & 0 & 0 & 9 & 0 & 0 \\
\hline 79 & $\mathrm{CF}_{3} \mathrm{CFHCFHCF}_{3}$ & $\begin{array}{l}1,1,1,2,3,4,4,4- \\
\text { Octafluorobutane }\end{array}$ & -14.34 & 2810 & 2.87 & 202.046 & 0 & 0 & 2 & 2 & 0 & 0 & 0 & 0 & 8 & 0 & 0 \\
\hline 80 & $\mathrm{CF}_{2} \mathrm{HCF}_{2} \mathrm{CF}_{2} \mathrm{CF}_{2} \mathrm{H}$ & $\begin{array}{l}1,1,2,2,3,3,4,4- \\
\text { Octafluorobutane }\end{array}$ & -14.34 & 2810 & 2.87 & 202.046 & 0 & 0 & 2 & 2 & 0 & 0 & 0 & 0 & 8 & 0 & 0 \\
\hline 82 & Fluorobenzene & & 84.7 & 77.2 & 2.27 & 96.1023 & 0 & 0 & 0 & 0 & 0 & 0 & 0 & 0 & 1 & 1 & 5 \\
\hline
\end{tabular}




\begin{tabular}{|c|c|c|c|c|c|c|c|c|c|c|c|c|c|c|c|c|c|}
\hline 83 & o-Difluorobenzene & 1,2-difluorobenzene & 94 & 53.4 & 2.37 & 114.0928 & 0 & 0 & 0 & 0 & 0 & 0 & 0 & 0 & 2 & 1 & 4 \\
\hline 84 & p-Difluorobenzene ${ }^{* *}$ & 1,4-difluorobenzene & 90.4 & 63.6 & 2.39 & 114.0928 & 0 & 0 & 0 & 0 & 0 & 0 & 0 & 0 & 2 & 1 & 4 \\
\hline 85 & 1,2,4-Trifluorobenzene & & 90 & 57.4 & 2.52 & 132.083 & 0 & 0 & 0 & 0 & 0 & 0 & 0 & 0 & 3 & 1 & 3 \\
\hline 86 & 1,3,5-Trifluorobenzene ${ }^{* *}$ & & 75.4 & 115.7 & 2.49 & 132.083 & 0 & 0 & 0 & 0 & 0 & 0 & 0 & 0 & 3 & 1 & 3 \\
\hline 87 & Pentafluorobenzene & & 85.7 & 67.6 & 2.53 & 168.0642 & 0 & 0 & 0 & 0 & 0 & 0 & 0 & 0 & 5 & 1 & 1 \\
\hline 88 & Hexafluorobenzene & Perfluorobenzene & 80.2 & 84.5 & 2.55 & 186.0546 & 0 & 0 & 0 & 0 & 0 & 0 & 0 & 0 & 6 & 1 & 0 \\
\hline 89 & Methylpentafluorobenzene & $\begin{array}{l}\text { Pentafluorotoluene, } \\
2,3,4,5,6 \text {-Pentafluorotoluene }\end{array}$ & 117.5 & 17.5 & 3.54 & 182.0907 & 0 & 0 & 0 & 0 & 0 & 0 & 0 & 0 & 5 & 1 & 0 \\
\hline 90 & $\mathrm{CBrClH}_{2}$ & Bromochloromethane & 68 & 143 & 1.41 & 129.3836 & 0 & 1 & 0 & 0 & 0 & 0 & 1 & 1 & 0 & 0 & 0 \\
\hline 91 & $\mathrm{CBr}_{2} \mathrm{ClH}$ & $\begin{array}{l}\text { Dibromochloromethane, } \\
\text { Chlorodibromomethane }\end{array}$ & 120 & 15.6 & 2.16 & 208.2796 & 0 & 0 & 1 & 0 & 0 & 0 & 2 & 1 & 0 & 0 & 0 \\
\hline 92 & $\mathrm{CClFH}_{2}$ & \begin{tabular}{|l|} 
Chlorofluoromethane, \\
Fluorochloromethane
\end{tabular} & -9.1 & 2450 & 0.51 & 68.47798 & 0 & 1 & 0 & 0 & 0 & 0 & 0 & 1 & 1 & 0 & 0 \\
\hline 93 & $\mathrm{CBrH}_{2} \mathrm{CClH}_{2}$ & 1-Bromo-2-chloroethane & 107 & 33.1 & 1.92 & 143.4102 & 0 & 2 & 0 & 0 & 0 & 0 & 1 & 1 & 0 & 0 & 0 \\
\hline 94 & $\mathrm{CF}_{3} \mathrm{CClH}_{2}$ & $\begin{array}{l}\text { F 133a, 2-Chloro-1,1,1- } \\
\text { trifluoroethane, 1,1,1- } \\
\text { Trifluoro-2-chloroethane }\end{array}$ & 6.1 & 1500 & 1.99 & 118.4855 & 0 & 1 & 0 & 0 & 1 & 0 & 0 & 1 & 3 & 0 & 0 \\
\hline 95 & Halothane & $\begin{array}{l}\text { CF3CBrClH, 1,1,1- } \\
\text { Trifluoro-2-bromo-2- } \\
\text { chloroethane }\end{array}$ & 50.2 & 302 & 2.3 & 197.3816 & 0 & 0 & 1 & 1 & 0 & 0 & 1 & 1 & 3 & 0 & 0 \\
\hline 96 & Teflurane & $\begin{array}{l}\text { CF3CBrFH, 2-Bromo- } \\
\text { 1,1,1,2-tetrafluoroethane }\end{array}$ & 8.6 & 1340 & 1.95 & 180.927 & 0 & 0 & 1 & 1 & 0 & 0 & 1 & 0 & 4 & 0 & 0 \\
\hline
\end{tabular}

* The QSAR Method described in ${ }^{4}$ and ${ }^{2}$ does not account for nitro, hydroxyl, ether, acetate or ketone groups which are included in the volatile organic carbon (VOC) chemicals given by ${ }^{1}$. However, the gasoline vapor condensate studied in this research does not include any of these chemicals, except for Ethanol and Methanol. Therefor to accurately compare the PC estimation methods, we excluded the nitro compounds, alcohols, ethers, acetates and ketones (chemical numbers 69,70, and 97 through 137). We also excluded chemical $81, \mathrm{CF}_{2} \mathrm{HCFHCH}_{2} \mathrm{CF}_{2} \mathrm{H}$ because we could not find accurate $\mathrm{BP}$, VP, or log Kow data for this chemical formula.

** All of the data in this table was taken from ChemSpider ${ }^{5}$ and the EPISuite tool ${ }^{6}$. If the experimental data was not available the estimated values for BP, VP, or log Kow were used. For chemicals 84 and 85, p-Difluorobenzene and 1,3,5-Trifluorobenzene, no EPISuite information was available, so the ACD Labs PhysChem Module estimates on ChemSpider were used.

Chemical names in bold indicate chemicals that were present in the gasoline vapor condensate used in this research. 


\section{Supplemental Table 3}

\section{Comparison of Rat PC Data with Human PC Data and PC Estimation Methods for Select ${ }^{*}$ Meulenberg Chemicals using log P for Blood, Liver, Fat, and Muscle to Air}

\begin{tabular}{|c|c|c|c|c|}
\hline & $\begin{array}{l}\text { Human PC Data } \\
\text { Meulenberg } 2000\end{array}$ & $\begin{array}{c}\text { QSAR Method } \\
\text { Beliveau } 2003 \\
\text { Price } 2011\end{array}$ & $\begin{array}{c}\text { Kow Method } \\
\text { Poulin } 1995 \\
\text { El-Masri } 1998\end{array}$ & $\begin{array}{c}\text { BP Method } \\
\text { Perbellini } 1985\end{array}$ \\
\hline \multicolumn{5}{|l|}{ Rat PC Data for Meulenberg Chemicals } \\
\hline Sum Squared Error (SSE) & 4.3433 & 118.1719 & 174.2084 & 128.0236 \\
\hline Root Mean Squared Error (RMSE) & 0.2360 & 0.7363 & 0.8939 & 0.8082 \\
\hline \multicolumn{5}{|c|}{ Rat PC Data for Meulenberg Chemicals in Gasoline Vapor Condensate ${ }^{* *}$} \\
\hline Sum Squared Error (SSE) & 1.8193 & 34.7179 & 54.9898 & 10.1735 \\
\hline Root Mean Squared Error (RMSE) & 0.2505 & 0.8505 & 1.0703 & 0.4604 \\
\hline \multicolumn{5}{|c|}{ Rat and Human PC Data ${ }^{* * *}$ for Meulenberg Chemicals } \\
\hline Sum Squared Error (SSE) & & 142.3321 & 260.3208 & 150.6065 \\
\hline Root Mean Squared Error (RMSE) & & 0.7207 & 0.9747 & 0.8218 \\
\hline \multicolumn{5}{|c|}{ Rat and Human PC Data for Meulenberg Chemicals in Gasoline Vapor Condensate } \\
\hline Sum Squared Error (SSE) & & 40.8823 & 119.1026 & 11.6383 \\
\hline Root Mean Squared Error (RMSE) & & 0.7287 & 1.2437 & 0.3888 \\
\hline
\end{tabular}

* See note on Supplemental Table 3 for excluded chemicals.

** The chemicals indicated in bold in Supplemental Table 3.

*** Since the Human PC data is closer to the Rat PC data than any of the estimation methods (as seen in Supplemental Tables 3 and 4 ), the Rat and Human can be combined as is done in other studies ${ }^{7}$. We used the rat data when available, and human PC data if no rat data was available.

In Supplemental Table 4 we show four sets of comparisons of the rat partition coefficient (PC) data in Meulenberg and Vijverberg ${ }^{1}$ against the human PC data compiled by Meulenberg et al. and other PC estimation methods. These estimation methods were chosen because of their widespread use and their ease of use. The PC method that Meulenberg presented in this paper is not considered because it requires experimental oil:air and saline:air partition coefficients which are not readily available. Although the Perbellini, et al. ${ }^{3}$ method actually estimates human PC values, it does a good job of estimating rat PC values. It was modified to take the maximum of the tissue:air PC value and the blood:air PC value for each tissue, because for low boiling points, the Perbellini et al. method would give negative PC values. Furthermore, it has been shown Abraham, et al. ${ }^{7}$ that the differences between the rat and human blood:air PC values are less than inter-laboratory experimental error. Our comparisons indicated that the average difference between rat and human PC data for all tissue values reported by Meulenberg are around $0.25 \log$ units.

For each these comparisons we display the sum squared error (SSE), that is the sum of the squared differences between the rat PC data and the human or estimated PC values, and the root mean squared error (RMSE) that gives the average difference between the PC data and the estimation methods. For the first two sets of comparisons, we use rat PC data and compare against human PC data and the PC estimation methods, in the last two sets of comparisons, we use combined rat and human PC data. We use the rat PC data first, if it is available, but if the rat PC data is not available and human PC data is, we use human PC data instead.

Finally, we chose to use the boiling point PC estimation method by Perbellini, et al. ${ }^{3}$. This is because for the gasoline chemicals for which there is PC data, it does the best job of estimating the PC values. Even though for all select Meulenberg chemicals the QSAR method does a slightly better job, the BP method is not far different. Also the QSAR method has the limitation that it does not consider nitro, hydroxyl, ether, acetate or ketone groups, and does not estimate the brain or kidney partition coefficients. The BP method of Perbellini, et al. ${ }^{3}$ is able to estimate the PC values for the blood, fat, brain, liver, muscle, kidney, heart and lung, and is not limited by functional groups. 
Supplemental Table 4

Chemical Specific Parameters for All 109 Known Measurable Chemicals in Gasoline Sample

\begin{tabular}{|c|c|c|c|c|c|c|c|c|c|c|c|c|c|c|}
\hline No. & Name & Percent & ppmv & MW & BP & PBA & PFat & PBrn & PLiv & PMsl & PKid & PLng & $V \max C L$ & KmL \\
\hline 1 & Isopentane & 36.459229 & 3269.458 & 72.15 & 27.8 & 0.19 & 158.81 & 11.28 & 16.22 & 3.88 & 4.14 & $\underline{0.03}$ & 0.26 & 0.35 \\
\hline 2 & n-Butane & 17.979411 & 2015.283 & 58.12 & -0.5 & 0.29 & 25.47 & 4.83 & 8.35 & 0.27 & 0.72 & 1.06 & 0.18 & 0.23 \\
\hline 3 & n-Pentane & 9.339235 & 837.517 & 72.15 & 36.06 & 0.38 & 104.21 & 5.79 & 5.53 & 1.84 & 1.58 & $\underline{0.05}$ & 0.29 & 0.40 \\
\hline 4 & 2-Methylpentane & 3.793963 & 283.537 & 86.18 & 60.21 & 0.41 & 212.20 & 9.27 & 10.98 & 7.07 & 4.88 & $\underline{0.20}$ & 0.28 & 1.02 \\
\hline 5 & Isobutane & 3.346761 & 375.134 & 58.12 & -11.7 & 0.06 & 49.07 & 22.63 & 41.45 & 1.00 & 1.62 & 4.98 & 0.16 & 0.20 \\
\hline 6 & 2-Methyl-2-Butene & 2.296306 & 205.922 & 70.13 & 38.5 & 0.26 & 171.78 & 9.94 & 13.24 & 4.93 & 4.44 & 2.16 & 0.06 & 0.43 \\
\hline 7 & Toluene & 2.178474 & 139.536 & 92.14 & 110.6 & 15.90 & 62.26 & 2.29 & 5.26 & 1.74 & 1.12 & $\underline{0.79}$ & 3.44 & 0.13 \\
\hline 8 & 3-Methylpentane & 2.078420 & 155.328 & 86.18 & 63.271 & 0.43 & 237.21 & 10.23 & 11.40 & 8.84 & 5.81 & 2.29 & 0.39 & 0.59 \\
\hline 9 & 2,3-Dimethylbutane & 1.944439 & 145.315 & 100.20 & 57.9 & 0.48 & 186.72 & 8.39 & 9.80 & 6.13 & 4.78 & 1.82 & 0.41 & 0.60 \\
\hline 10 & 2,2,4-TriMethylpentane & 1.928256 & 108.065 & 114.23 & 99.238 & 1.77 & 165.54 & 6.75 & 6.05 & 1.86 & 4.88 & 1.46 & 0.03 & 0.82 \\
\hline 11 & n-Hexane & 1.796269 & 134.235 & 86.18 & 68.73 & 1.72 & 92.44 & 2.91 & 3.02 & 1.69 & 1.74 & $\underline{0.14}$ & 1.27 & 3.65 \\
\hline 12 & trans-2-Pentene & 1.635991 & 146.702 & 70.13 & 36.3 & 0.24 & 169.45 & 10.18 & 13.77 & 4.74 & 4.38 & 2.22 & 0.20 & 0.34 \\
\hline 13 & Methylcyclopentane & 1.188279 & 88.807 & 84.16 & 71.8 & 0.86 & 204.65 & 8.49 & 9.07 & 5.81 & 5.47 & 1.42 & 0.32 & 0.61 \\
\hline 14 & Cyclohexane & 0.924894 & 69.124 & 84.16 & 80.7 & 1.39 & 169.06 & 7.70 & 5.67 & 0.74 & 5.18 & $\underline{0.21}$ & 3.79 & 5.81 \\
\hline 15 & 2,2-Dimethylbutane & 0.923719 & 69.033 & 86.18 & 49.7 & 0.26 & 253.85 & 10.77 & 13.46 & 3.85 & 5.38 & 2.76 & 0.01 & 0.35 \\
\hline 16 & 2-Methyl-1-Butene & 0.914738 & 82.026 & 70.13 & 31.2 & 0.21 & 163.40 & 10.80 & 15.16 & 4.25 & 4.24 & 2.36 & 0.20 & 0.34 \\
\hline 17 & 2,4-Dimethylpentane & 0.818756 & 52.446 & 100.20 & 80.4 & 0.95 & 195.86 & 7.45 & 7.70 & 6.86 & 4.99 & 1.61 & 0.52 & 0.85 \\
\hline 18 & Cyclopentane & 0.780254 & 69.973 & 70.13 & 49.2 & 1.74 & 37.90 & 1.88 & 2.32 & 1.19 & 0.97 & 0.41 & 0.23 & 0.41 \\
\hline 19 & 3-Methylhexane & 0.727727 & 46.613 & 100.20 & 91 & 1.30 & 213.08 & 7.85 & 8.15 & 8.31 & 5.62 & 1.57 & 0.58 & 0.98 \\
\hline 20 & 2,3-Dimethylpentane & 0.725218 & 46.451 & 100.20 & 89.7 & 1.27 & 198.14 & 7.21 & 7.18 & 7.05 & 5.04 & 1.56 & 0.52 & 0.85 \\
\hline 21 & cis-2-Pentene & 0.705509 & 63.262 & 70.13 & 36.9 & 0.25 & 170.10 & 10.11 & 13.62 & 4.79 & 4.40 & 2.20 & 0.20 & 0.34 \\
\hline 22 & 2-Methylhexane & 0.690698 & 44.243 & 100.20 & 90 & 1.28 & 198.20 & 7.20 & 7.16 & 7.05 & 5.04 & 1.56 & 0.48 & 1.68 \\
\hline 23 & Benzene & 0.640876 & 47.908 & 78.11 & 80.08 & 14.10 & 30.67 & 1.28 & 1.21 & 0.73 & 0.86 & $\underline{0.73}$ & 2.11 & 0.10 \\
\hline 24 & n-Heptane & 0.459966 & 29.459 & 100.20 & 98.38 & 4.75 & 79.79 & 2.61 & 3.16 & 0.88 & 1.87 & 0.53 & 1.24 & 2.36 \\
\hline 25 & 1-Pentene & 0.357771 & 32.081 & 70.13 & 29.9 & 0.20 & 161.70 & 10.98 & 15.55 & 4.12 & 4.20 & 2.40 & 0.73 & 0.27 \\
\hline
\end{tabular}




\begin{tabular}{|c|c|c|c|c|c|c|c|c|c|c|c|c|c|c|}
\hline 26 & 2,3,4-TriMethylpentane & 0.268497 & 15.046 & 114.23 & 113.5 & 3.75 & 118.13 & 4.81 & 5.01 & 1.18 & 3.60 & 1.04 & 0.67 & 1.20 \\
\hline 27 & 2-Methyl-2-Pentene & 0.259033 & 19.358 & 84.16 & 67.3 & 0.64 & 191.32 & 7.92 & 8.75 & 6.50 & 4.89 & 1.71 & 0.33 & 0.57 \\
\hline 28 & Propane & 0.238329 & 35.681 & 44.10 & -42.1 & 0.02 & 1.00 & 47.64 & 97.03 & 1.00 & 1.00 & 10.54 & 0.11 & 0.12 \\
\hline 29 & trans-2-Hexene & 0.208686 & 15.594 & 84.16 & 65 & 0.59 & 190.31 & 8.02 & 8.98 & 6.42 & 4.86 & 1.74 & 0.31 & 0.58 \\
\hline 30 & trans-3-Methyl-2-pentene & 0.207339 & 15.495 & 84.16 & 67.7 & 0.64 & 191.48 & 7.90 & 8.71 & 6.51 & 4.89 & 1.71 & 0.33 & 0.57 \\
\hline 31 & trans-2-Butene & 0.201197 & 22.547 & 56.11 & 0.8 & 0.08 & 98.90 & 17.48 & 29.99 & 1.00 & 2.76 & 3.84 & 0.13 & 0.19 \\
\hline 32 & m-Xylene & 0.197904 & 11.092 & 106.17 & 139.07 & 46.00 & 45.48 & 0.84 & 1.98 & 0.91 & 0.65 & $\underline{0.56}$ & 6.49 & 0.45 \\
\hline 33 & Cyclopentene & 0.182454 & 16.362 & 68.12 & 44.2 & 0.31 & 177.13 & 9.38 & 12.01 & 5.36 & 4.56 & 2.04 & 0.16 & 0.34 \\
\hline 34 & MethylCyclohexane & 0.179721 & 11.511 & 98.19 & 100.9 & 1.79 & 200.14 & 7.00 & 6.72 & 7.21 & 5.09 & 1.51 & 1.61 & 3.46 \\
\hline 35 & Ethanol & 0.171320 & 38.373 & 46.07 & 78.29 & 2355.00 & 0.10 & 0.79 & 0.73 & 0.73 & 0.86 & 0.00 & 272.00 & 23.00 \\
\hline 36 & cis-2-Butene & 0.159434 & 17.870 & 56.11 & 3.7 & 0.09 & 107.96 & 16.54 & 27.91 & 1.00 & 2.97 & 3.63 & 0.13 & 0.19 \\
\hline 37 & cis-3-Methyl-2-pentene & 0.142922 & 10.681 & 84.16 & 67.7 & 0.64 & 191.48 & 7.90 & 8.71 & 6.51 & 4.89 & 1.71 & 0.08 & 0.73 \\
\hline 38 & 2-Methyl-1-pentene & 0.129916 & 9.713 & 84.16 & 62.1 & 0.54 & 188.94 & 8.16 & 9.29 & 6.31 & 4.83 & 1.77 & 0.31 & 0.58 \\
\hline 39 & cis-3-Hexene & 0.127707 & 9.543 & 84.16 & 67.1 & 0.63 & 191.23 & 7.92 & 8.77 & 6.49 & 4.88 & 1.72 & 0.31 & 0.58 \\
\hline 40 & 2,2,5-Trimethylhexane & 0.124600 & 6.207 & 128.26 & 124 & 3.65 & 202.61 & 6.75 & 6.15 & 7.41 & 5.15 & 1.45 & 0.03 & 1.16 \\
\hline 41 & 3-Methyl-1-Butene & 0.124134 & 11.132 & 70.13 & 20.1 & 0.15 & 146.44 & 12.56 & 19.06 & 2.89 & 3.85 & 2.75 & 0.65 & 0.23 \\
\hline 42 & cis-1,3-Dimethylcyclopentane & 0.108383 & 6.942 & 98.19 & 90.8 & 1.31 & 198.36 & 7.19 & 7.13 & 7.07 & 5.05 & 1.55 & 0.43 & 0.87 \\
\hline 43 & 1-Methylcyclopentene & 0.108228 & 8.071 & 82.14 & 75.5 & 0.82 & 194.37 & 7.60 & 8.04 & 6.74 & 4.96 & 1.64 & 0.07 & 0.75 \\
\hline 44 & 2,5-Dimethylhexane & 0.104413 & 5.851 & 114.23 & 109.1 & 2.30 & 201.22 & 6.89 & 6.47 & 7.30 & 5.11 & 1.49 & 0.75 & 1.38 \\
\hline 45 & 3-Methylheptane & 0.104130 & 5.837 & 114.23 & 118 & 3.03 & 202.13 & 6.80 & 6.26 & 7.37 & 5.13 & 1.47 & 0.84 & 1.59 \\
\hline 46 & Ethylbenzene & 0.103315 & 5.790 & 106.17 & 136.1 & 30.45 & 51.10 & 1.16 & 1.98 & 0.85 & 0.90 & $\underline{0.74}$ & 4.58 & 0.52 \\
\hline 47 & p-Xylene & 0.099696 & 5.588 & 106.17 & 138.23 & 41.30 & 42.32 & 0.91 & 2.18 & 0.93 & 0.71 & $\underline{0.57}$ & 5.31 & 0.72 \\
\hline 48 & 2,4-Dimethylhexane & 0.093669 & 5.249 & 114.23 & 109.5 & 2.33 & 201.27 & 6.89 & 6.46 & 7.30 & 5.11 & 1.49 & 0.75 & 1.38 \\
\hline 49 & Ethylcyclopentane & 0.093669 & 5.249 & 98.19 & 103.5 & 1.94 & 200.51 & 6.96 & 6.63 & 7.24 & 5.10 & 1.50 & 0.48 & 1.00 \\
\hline 50 & cis-2-Hexene & 0.092503 & 6.912 & 84.16 & 65 & 0.59 & 190.31 & 8.02 & 8.98 & 6.42 & 4.86 & 1.74 & 0.31 & 0.58 \\
\hline 51 & o-Xylene & 0.092195 & 5.172 & 106.17 & 144.5 & 44.30 & 54.27 & 1.03 & 2.44 & 1.16 & 0.80 & $\underline{0.70}$ & 5.31 & 0.72 \\
\hline 52 & 2,2-Dimethylpropane & 0.090612 & 8.127 & 72.15 & 9.5 & 0.11 & 123.84 & 14.90 & 24.26 & 1.07 & 3.33 & 3.27 & 0.01 & 0.21 \\
\hline 53 & 3-EthylPentane & 0.083085 & 5.322 & 98.19 & 90.8 & 1.31 & 198.36 & 7.19 & 7.13 & 7.07 & 5.05 & 1.55 & 0.43 & 0.87 \\
\hline 54 & trans-1,3-DiMethylCyclopentane & 0.083085 & 5.322 & 100.20 & 93.5 & 1.42 & 198.89 & 7.13 & 7.00 & 7.11 & 5.06 & 1.54 & 0.58 & 0.98 \\
\hline 55 & 3-Methyl-cis-3-Hexene & 0.081653 & 5.230 & 98.19 & 112.5 & 2.56 & 201.60 & 6.85 & 6.38 & 7.33 & 5.12 & 1.48 & 0.13 & 1.22 \\
\hline
\end{tabular}




\begin{tabular}{|c|c|c|c|c|c|c|c|c|c|c|c|c|c|c|}
\hline 56 & 2-methylheptane & 0.080284 & 4.500 & 114.23 & 116 & 2.85 & 201.95 & 6.82 & 6.30 & 7.35 & 5.13 & 1.47 & 0.84 & 1.59 \\
\hline 57 & 3,3-Dimethylpentane & 0.077599 & 4.971 & 100.20 & 86 & 1.13 & 197.31 & 7.30 & 7.37 & 6.98 & 5.02 & 1.58 & 0.02 & 0.58 \\
\hline 58 & 2,3-Dimethylhexane & 0.070527 & 3.953 & 114.23 & 115.6 & 2.81 & 201.91 & 6.82 & 6.31 & 7.35 & 5.13 & 1.47 & 0.75 & 1.38 \\
\hline 59 & 2,2-Dimethylpentane & 0.070143 & 4.493 & 100.20 & 79.2 & 0.92 & 195.52 & 7.48 & 7.78 & 6.84 & 4.98 & 1.62 & 0.02 & 0.58 \\
\hline 60 & 3-Methyl-1-pentene & 0.053990 & 4.035 & 84.16 & 54.2 & 0.42 & 184.52 & 8.62 & 10.31 & 5.95 & 4.73 & 1.87 & 1.00 & 0.40 \\
\hline 61 & n-octane & 0.053009 & 2.971 & 114.23 & 125.62 & 7.53 & 30.94 & 2.19 & 3.43 & 1.14 & 1.09 & 0.74 & 0.71 & 1.19 \\
\hline 62 & Isobutylene & 0.052054 & 5.834 & 56.11 & -6.9 & 0.06 & 70.50 & 20.41 & 36.52 & 1.00 & 2.11 & 4.49 & 0.13 & 0.19 \\
\hline 63 & 4-Methylheptane & 0.040368 & 2.263 & 114.23 & 117.7 & 3.00 & 202.10 & 6.80 & 6.27 & 7.37 & 5.13 & 1.47 & 0.84 & 1.59 \\
\hline 64 & 1,1-Dimethylcyclopentane & 0.034630 & 2.218 & 98.19 & 87.5 & 1.18 & 197.65 & 7.26 & 7.29 & 7.01 & 5.03 & 1.57 & 0.02 & 0.60 \\
\hline 65 & 1-hexene & 0.031248 & 2.334 & 84.16 & 63.4 & 0.56 & 189.57 & 8.10 & 9.15 & 6.36 & 4.85 & 1.75 & $\mathbf{0 . 5 7}$ & 1.11 \\
\hline 66 & 2,2,3-TriMethylbutane & 0.028872 & 1.850 & 100.20 & 80.8 & 0.96 & 195.97 & 7.43 & 7.68 & 6.87 & 4.99 & 1.61 & 0.02 & 0.50 \\
\hline 67 & 1-Butene & 0.026165 & 2.934 & 56.11 & -6.47 & 0.07 & 72.27 & 20.23 & 36.11 & 1.00 & 2.15 & 4.45 & 0.46 & 0.15 \\
\hline 68 & 1,2,4-trimethylbenzene & 0.024129 & 1.202 & 120.19 & 168.89 & 14.53 & 204.40 & 6.56 & 5.74 & 7.55 & 5.19 & 1.41 & 8.71 & 1.72 \\
\hline 69 & trans-3-Heptene & 0.021996 & 1.409 & 98.19 & 95.7 & 1.52 & 199.29 & 7.09 & 6.91 & 7.14 & 5.07 & 1.53 & 0.46 & 0.96 \\
\hline 70 & trans-3-Methyl-2-hexene & 0.021935 & 1.405 & 98.19 & 95.6 & 1.52 & 199.28 & 7.09 & 6.92 & 7.14 & 5.07 & 1.53 & 0.13 & 1.22 \\
\hline 71 & 3,4-DiMethylhexane & 0.018632 & 1.045 & 114.23 & 117.7 & 3.00 & 202.10 & 6.80 & 6.27 & 7.37 & 5.13 & 1.47 & 0.75 & 1.38 \\
\hline 72 & m-Ethyltoluene & 0.018498 & 0.922 & 120.19 & 161.3 & 11.50 & 204.24 & 6.58 & 5.77 & 7.54 & 5.18 & 1.42 & 7.65 & 1.16 \\
\hline 73 & ctc-1,2,4-TriMethylCyclopentane & 0.016455 & 0.923 & 112.21 & 109.3 & 2.32 & 201.25 & 6.89 & 6.46 & 7.30 & 5.11 & 1.49 & 0.56 & 1.23 \\
\hline 74 & 2,3-Dimethyl-2-pentene & 0.015781 & 1.011 & 98.19 & 97.5 & 1.61 & 199.60 & 7.06 & 6.84 & 7.17 & 5.08 & 1.52 & 0.03 & 1.54 \\
\hline 75 & 2,3,5-TriMethylhexane & 0.015682 & 0.781 & 128.26 & 131.4 & 4.58 & 203.10 & 6.70 & 6.04 & 7.45 & 5.16 & 1.44 & 0.96 & 1.91 \\
\hline 76 & 2,4,4-TriMethyl-1-Pentene & 0.015112 & 0.847 & 112.21 & 101.4 & 1.82 & 200.21 & 7.00 & 6.70 & 7.21 & 5.09 & 1.51 & 0.02 & 0.80 \\
\hline 77 & 2,5-DiMethylHeptane & 0.013735 & 0.684 & 128.26 & 136 & 5.28 & 203.35 & 6.67 & 5.98 & 7.47 & 5.16 & 1.44 & 1.07 & 2.20 \\
\hline 78 & 3,3-DiMethylhexane & 0.013561 & 0.760 & 114.23 & 111.9 & 2.51 & 201.54 & 6.86 & 6.40 & 7.32 & 5.12 & 1.48 & 0.03 & 0.94 \\
\hline 79 & 1,2,4-TriMethylCyclopentane & 0.013561 & 0.760 & 112.21 & 109.3 & 2.32 & 201.25 & 6.89 & 6.46 & 7.30 & 5.11 & 1.49 & 0.56 & 1.23 \\
\hline 80 & Methanol & 0.013192 & 5.992 & 32.04 & 64.7 & 3335.00 & 0.06 & 1.04 & 0.93 & 1.19 & 0.96 & 0.00 & 30.00 & 224.29 \\
\hline 81 & cis-4-Methyl-2-hexene & 0.011903 & 0.763 & 98.19 & 87.6 & 1.19 & 197.68 & 7.26 & 7.28 & 7.01 & 5.03 & 1.57 & 0.41 & 0.83 \\
\hline 82 & 2,2,4-TriMethylhexane & 0.011646 & 0.580 & 128.26 & 126.5 & 3.94 & 202.79 & 6.73 & 6.11 & 7.42 & 5.15 & 1.45 & 0.04 & 1.31 \\
\hline 83 & ctc-1,2,3-TriMethylCyclopentane & 0.011021 & 0.618 & 112.21 & 117.5 & 2.98 & 202.08 & 6.80 & 6.27 & 7.37 & 5.13 & 1.47 & 0.56 & 1.23 \\
\hline 84 & 1,3,5-TriMethylbenzene & 0.008672 & 0.432 & 120.19 & 164.7 & 43.00 & 60.67 & 1.95 & 1.71 & 2.24 & 1.54 & 0.42 & 8.87 & 1.60 \\
\hline 85 & p-Ethyltoluene & 0.008552 & 0.427 & 120.19 & 162 & 11.75 & 204.26 & 6.58 & 5.77 & 7.54 & 5.18 & 1.42 & 7.65 & 1.16 \\
\hline
\end{tabular}




\begin{tabular}{|c|c|c|c|c|c|c|c|c|c|c|c|c|c|c|}
\hline 86 & 3-Methyloctane & 0.007722 & 0.385 & 128.26 & 144.2 & 6.79 & 203.72 & 6.63 & 5.89 & 7.50 & 5.17 & 1.43 & 1.20 & 2.54 \\
\hline 87 & 2-Methyl-3-Ethylpentane & 0.007226 & 0.405 & 114.23 & 115.6 & 2.81 & 201.91 & 6.82 & 6.31 & 7.35 & 5.13 & 1.47 & 0.75 & 1.38 \\
\hline 88 & 2,4-DiMethylheptane & 0.006835 & 0.341 & 128.26 & 132.9 & 4.80 & 203.19 & 6.69 & 6.02 & 7.45 & 5.16 & 1.44 & 1.07 & 2.20 \\
\hline 89 & n-Propylbenzene & 0.006783 & 0.339 & 120.19 & 159.2 & 10.78 & 204.19 & 6.58 & 5.79 & 7.54 & 5.18 & 1.42 & 6.61 & 0.84 \\
\hline 90 & 4-Methyl-1-Hexene & 0.006553 & 0.420 & 98.19 & 86.7 & 1.16 & 197.47 & 7.28 & 7.33 & 6.99 & 5.03 & 1.57 & 1.42 & 0.52 \\
\hline 91 & Propylene & 0.005774 & 1.294 & 42.08 & -48 & 0.02 & 1.00 & 55.84 & 115.25 & 1.00 & 1.00 & 12.36 & 0.27 & 0.08 \\
\hline 92 & o-Ethyltoluene & 0.005629 & 0.281 & 120.19 & 165.2 & 12.97 & 204.33 & 6.57 & 5.75 & 7.55 & 5.18 & 1.42 & 7.65 & 1.16 \\
\hline 93 & 2,4-DiMethyl-1-Pentene & 0.005337 & 0.342 & 98.19 & 81.6 & 0.99 & 196.19 & 7.41 & 7.63 & 6.89 & 5.00 & 1.60 & 0.41 & 0.83 \\
\hline 94 & ctt-1,2,4-TriMethylCyclohexane & 0.004933 & 0.246 & 126.24 & 142.9 & 6.52 & 203.67 & 6.64 & 5.91 & 7.49 & 5.17 & 1.43 & 0.80 & 1.97 \\
\hline 95 & 1,2,3-TriMethylbenzene & 0.004906 & 0.244 & 120.19 & 176.12 & 66.50 & 55.83 & 1.79 & 1.56 & 2.06 & 1.42 & 0.39 & 8.87 & 1.60 \\
\hline 96 & trans-1,4-DiMethylCyclohexane & 0.004876 & 0.274 & 112.21 & 119.4 & 3.16 & 202.25 & 6.78 & 6.23 & 7.38 & 5.14 & 1.46 & 0.62 & 1.42 \\
\hline 97 & 2,6-DiMethylheptane & 0.004336 & 0.216 & 128.26 & 135.2 & 5.15 & 203.31 & 6.67 & 5.99 & 7.46 & 5.16 & 1.44 & 1.07 & 2.20 \\
\hline 98 & n-nonane & 0.004227 & 0.211 & 128.26 & 150.47 & 8.24 & 203.94 & 6.61 & 5.84 & 7.52 & 5.18 & 1.42 & 0.59 & 0.89 \\
\hline 99 & cis-2-Octene & 0.003836 & 0.213 & 112.21 & 125 & 3.76 & 202.69 & 6.74 & 6.13 & 7.41 & 5.15 & 1.45 & 0.66 & 1.56 \\
\hline 100 & trans-2-Octene & 0.002120 & 0.119 & 112.21 & 125 & 3.76 & 202.69 & 6.74 & 6.13 & 7.41 & 5.15 & 1.45 & 0.66 & 1.56 \\
\hline 101 & Isopropylcyclopentane & 0.002107 & 0.118 & 112.21 & 126.5 & 3.94 & 202.79 & 6.73 & 6.11 & 7.42 & 5.15 & 1.45 & 0.62 & 1.42 \\
\hline 102 & 1,1,4-TriMethylCyclohexane & 0.001858 & 0.093 & 126.24 & 134.3 & 5.01 & 203.26 & 6.68 & 6.00 & 7.46 & 5.16 & 1.44 & 0.03 & 1.35 \\
\hline 103 & Indane & 0.001700 & 0.085 & 118.18 & 177.9 & 19.17 & 204.55 & 6.55 & 5.70 & 7.56 & 5.19 & 1.41 & 8.19 & 1.11 \\
\hline 104 & 2,2-DiMethylHeptane & 0.001147 & 0.057 & 128.26 & 132.7 & 4.77 & 203.17 & 6.69 & 6.02 & 7.45 & 5.16 & 1.44 & 0.04 & 1.50 \\
\hline 105 & 4,4-DiMethylheptane & 0.000863 & 0.043 & 128.26 & 135.2 & 5.15 & 203.31 & 6.67 & 5.99 & 7.46 & 5.16 & 1.44 & 0.04 & 1.50 \\
\hline 106 & cis-1,2-DiMethylcyclohexane & 0.000786 & 0.039 & 112.21 & 123.5 & 3.59 & 202.58 & 6.75 & 6.16 & 7.40 & 5.14 & 1.46 & 0.62 & 1.42 \\
\hline 107 & n-Propylcyclopentane & 0.000786 & 0.039 & 112.21 & 131 & 4.52 & 203.08 & 6.70 & 6.04 & 7.45 & 5.16 & 1.44 & 0.70 & 1.63 \\
\hline 108 & n-decane & 0.000769 & 0.034 & 142.28 & 174.1 & 17.30 & 201.62 & 6.46 & 5.64 & 7.45 & 5.12 & 1.39 & 1.13 & 1.50 \\
\hline 109 & 3-Methylnonane & 0.000349 & 0.016 & 142.28 & 167.9 & 14.09 & 204.38 & 6.56 & 5.74 & 7.55 & 5.19 & 1.41 & 1.69 & 4.00 \\
\hline
\end{tabular}

Values in bold are experimental rat data from ${ }^{1,2}$. Values in bold and italics are experimental human data from ${ }^{1}$. Values in italics are estimated data using the BP method in ${ }^{8}$ for PC values and the QSPR method in ${ }^{2}$ for the Vmax and Km values. The underlined PLng values were optimized to fit experimental exposure data in this research. 


\section{Supplemental Table 5}

Experimental Blood Concentrations for Target Chemicals

\begin{tabular}{|c|c|c|c|c|c|c|c|c|c|c|c|}
\hline \multicolumn{2}{|c|}{ Group 9Kppm- C } & \multicolumn{10}{|c|}{ Blood Concentration [mg/L] } \\
\hline Time $[\mathrm{h}]$ & Sample & n-Pentane & 2-Methylpentane & Toluene & n-Hexane & Cyclohexane & Benzene & m-Xylene & Ethylbenzene & p-Xylene & o-Xylene \\
\hline 6.138 & $\mathrm{C} 01$ & 0.02172 & 0.01852 & 0.77187 & 0.01439 & 0.01206 & 0.22053 & 0.035945 & 0.04668 & 0.019355 & 0.03099 \\
\hline 4.008 & $\mathrm{C} 02$ & 0.05059 & 0.04866 & 0.96704 & 0.06805 & 0.02469 & 0.30274 & 0.037655 & 0.04736 & 0.020276 & 0.03049 \\
\hline 6.008 & $\mathrm{C} 03$ & 0.08018 & 0.06446 & 1.44635 & 0.07952 & 0.03032 & 0.44774 & 0.066671 & 0.08875 & 0.0359 & 0.05032 \\
\hline 6.508 & $\mathrm{C} 04$ & 0.00376 & 0.00597 & 0.41358 & 0.00388 & 0.00501 & 0.12086 & 0.013338 & 0.0182 & 0.007182 & 0.0159 \\
\hline 7.008 & $\mathrm{C} 05$ & 0.01278 & 0.00529 & 0.33006 & 0.00379 & 0.00443 & 0.07765 & 0.012831 & 0.01587 & 0.006909 & 0.01698 \\
\hline 6.258 & $\mathrm{C} 06$ & 0.00977 & 0.00978 & 0.87957 & 0.00674 & 0.0079 & 0.22146 & 0.033521 & 0.04681 & 0.01805 & 0.02975 \\
\hline 6.138 & $\mathrm{C} 07$ & 0.02089 & 0.02227 & 1.20604 & 0.04955 & 0.01773 & 0.32253 & 0.045247 & 0.05519 & 0.024364 & 0.0392 \\
\hline 4.008 & $\mathrm{C} 08$ & 0.15094 & 0.11669 & 2.19466 & 0.13885 & 0.06244 & 0.64522 & 0.090818 & 0.10812 & 0.048902 & 0.06641 \\
\hline 6.008 & $\mathrm{C} 09$ & 0.17332 & 0.12541 & 2.97247 & 0.15864 & 0.0682 & 0.83923 & 0.135837 & 0.16079 & 0.073143 & 0.10895 \\
\hline 6.508 & $\mathrm{C} 10$ & 0.00487 & 0.00851 & 0.60746 & 0.00567 & 0.00717 & 0.18139 & 0.030511 & 0.03291 & 0.016429 & 0.03296 \\
\hline 7.008 & $\mathrm{C} 11$ & 0.0007 & 0.00899 & 0.54843 & 0.00508 & 0.00564 & 0.15396 & 0.024135 & 0.02935 & 0.012996 & 0.02622 \\
\hline 6.258 & $\mathrm{C} 12$ & 0.00346 & 0.00893 & 1.22548 & 0.02925 & 0.00794 & 0.2731 & 0.062582 & 0.07036 & 0.033698 & 0.05221 \\
\hline 6.138 & $\mathrm{C} 13$ & 0.01331 & 0.01552 & 1.58358 & 0.01233 & 0.01415 & 0.38318 & 0.057545 & 0.07318 & 0.030986 & 0.04792 \\
\hline 4.008 & $\mathrm{C} 14$ & 0.1044 & 0.0925 & 1.87608 & 0.1035 & 0.0437 & 0.57947 & 0.082024 & 0.09804 & 0.044167 & 0.05896 \\
\hline 6.008 & $\mathrm{C} 15$ & 0.16924 & 0.1368 & 3.04679 & 0.15895 & 0.07327 & 0.91311 & 0.111241 & 0.13653 & 0.059899 & 0.0844 \\
\hline 6.508 & $\mathrm{C} 16$ & - & 0.00724 & 0.80356 & 0.0054 & 0.00621 & 0.21511 & 0.026228 & 0.03509 & 0.014123 & 0.02815 \\
\hline 7.008 & $\mathrm{C} 17$ & 0.02965 & 0.00863 & 0.49502 & 0.00644 & 0.00673 & 0.13676 & 0.016933 & 0.02078 & 0.009118 & 0.02269 \\
\hline 6.258 & $\mathrm{C} 18$ & 0.00984 & 0.01101 & 0.63105 & 0.0069 & 0.00849 & 0.18654 & 0.024739 & 0.02713 & 0.013321 & 0.03059 \\
\hline 6.138 & $\mathrm{C} 19$ & 0.01748 & 0.01743 & 1.60025 & 0.0138 & 0.01567 & 0.41863 & 0.042575 & 0.0616 & 0.022925 & 0.03586 \\
\hline 4.008 & $\mathrm{C} 20$ & 0.13727 & 0.10838 & 2.42034 & 0.13331 & 0.06421 & 0.81055 & 0.063908 & 0.09171 & 0.034412 & 0.0475 \\
\hline 6.008 & $\mathrm{C} 21$ & 0.19078 & 0.15132 & 2.61823 & 0.17669 & 0.09809 & 0.87452 & 0.088803 & 0.10397 & 0.047817 & 0.06899 \\
\hline 6.508 & $\mathrm{C} 22$ & 0.00915 & 0.01652 & 1.00176 & 0.01161 & 0.01454 & 0.2759 & 0.033917 & 0.04155 & 0.018263 & 0.03684 \\
\hline 7.008 & $\mathrm{C} 23$ & 0.04293 & 0.022 & 0.43168 & 0.00669 & 0.0067 & 0.11085 & 0.015802 & 0.02546 & 0.008509 & 0.01854 \\
\hline 6.258 & $\mathrm{C} 24$ & 0.00678 & 0.01303 & 1.42491 & 0.00995 & 0.01241 & 0.35458 & 0.056739 & 0.05921 & 0.030552 & 0.05513 \\
\hline
\end{tabular}




\section{Supplemental Table 6}

\section{Chemical Constituents of the 5 Lumps}

\section{Using k-means Clustering to Group the non-Target Chemicals}

\begin{tabular}{|c|c|}
\hline $\begin{array}{l}10 \text { Target } \\
\text { Chems }\end{array}$ & n-Pentane; 2-Methylpentane; Toluene; n-Hexane; Cyclohexane; Benzene; m-Xylene; Ethylbenzene; p-Xylene; o-Xylene \\
\hline Lump1 & $\begin{array}{l}\text { 2,2,5-Trimethylhexane; 1,2,4-trimethylbenzene; m-Ethyltoluene; 2,3,5-TriMethylhexane; 2,5-DiMethylHeptane; 2,2,4- } \\
\text { TriMethylhexane; p-Ethyltoluene; 3-Methyloctane; 2,4-DiMethylheptane; n-Propylbenzene; o-Ethyltoluene; ctt-1,2,4- } \\
\text { TriMethylCyclohexane; 2,6-DiMethylheptane; n-nonane; 1,1,4-TriMethylCyclohexane; Indane; 2,2-DiMethylHeptane; 4,4- } \\
\text { DiMethylheptane; n-decane; 3-Methylnonane }\end{array}$ \\
\hline Lump2 & $\begin{array}{l}\text { 3-Methylpentane; 2,3-Dimethylbutane; Methylcyclopentane; 2,2-Dimethylbutane; 2,4-Dimethylpentane; 3-Methylhexane ; 2,3- } \\
\text { Dimethylpentane; 2-Methylhexane; MethylCyclohexane; cis-1,3-Dimethylcyclopentane; 2,5-Dimethylhexane; 3-Methylheptane; } \\
\text { 2,4-Dimethylhexane; Ethylcyclopentane; 3-EthylPentane; trans-1,3-DiMethylCyclopentane; 3-Methyl-cis-3-Hexene; 2- } \\
\text { methylheptane; 3,3-Dimethylpentane; 2,3-Dimethylhexane; 2,2-Dimethylpentane; 4-Methylheptane; 1,1-Dimethylcyclopentane; } \\
\text { 2,2,3-TriMethylbutane; trans-3-Heptene; trans-3-Methyl-2-hexene; 3,4-DiMethylhexane; ctc-1,2,4-TriMethylCyclopentane; 2,3- } \\
\text { Dimethyl-2-pentene; 2,4,4-TriMethyl-1-Pentene; 3,3-DiMethylhexane; 1,2,4-TriMethylCyclopentane; cis-4-Methyl-2-hexene; } \\
\text { ctc-1,2,3-TriMethylCyclopentane; 2-Methyl-3-Ethylpentane; 4-Methyl-1-Hexene; 2,4-DiMethyl-1-Pentene; trans-1,4- } \\
\text { DiMethylCyclohexane; cis-2-Octene; trans-2-Octene; Isopropylcyclopentane; cis-1,2-DiMethylcyclohexane; n- } \\
\text { Propylcyclopentane }\end{array}$ \\
\hline Lump3 & $\begin{array}{l}\text { Isopentane; 2-Methyl-2-Butene; 2,2,4-TriMethylpentane; trans-2-Pentene; 2-Methyl-1-Butene; cis-2-Pentene; 1-Pentene; 2,3,4- } \\
\text { TriMethylpentane; 2-Methyl-2-Pentene; trans-2-Hexene; trans-3-Methyl-2-pentene; Cyclopentene; cis-3-Methyl-2-pentene; 2- } \\
\text { Methyl-1-pentene; cis-3-Hexene; 3-Methyl-1-Butene; 1-Methylcyclopentene; cis-2-Hexene; 2,2-Dimethylpropane; 3-Methyl-1- } \\
\text { pentene; 1-hexene }\end{array}$ \\
\hline Lump4 & $\begin{array}{l}\text { n-Butane; Isobutane; Cyclopentane; n-Heptane; Propane; trans-2-Butene; cis-2-Butene; n-octane; Isobutylene; 1-Butene; 1,3,5- } \\
\text { TriMethylbenzene; Propylene; 1,2,3-TriMethylbenzene }\end{array}$ \\
\hline Lump5 & Ethanol; Methanol \\
\hline
\end{tabular}




\section{Supplemental Table 7}

Chemical Parameters for the 5 Lumps

Using k-means Clustering to Group the non-Target Chemicals

\begin{tabular}{|c|c|c|c|c|c|c|c|c|c|c|c|c|}
\hline Name & Percent & ppmv & MW & PBA & PFat & PBrn & PLiv & PMsl & PKid & PLng & VmaxCL & $\mathrm{KmL}$ \\
\hline Lump1 & 0.26 & 13.15 & 126.94 & 8.80 & 203.57 & 6.63 & 5.89 & 7.49 & 5.17 & 1.43 & 2.81 & 1.67 \\
\hline Lump2 & 10.63 & 740.51 & 104.34 & 2.00 & 202.29 & 7.26 & 7.16 & 7.13 & 5.12 & 1.57 & 0.50 & 1.14 \\
\hline Lump3 & 46.33 & 4059.92 & 81.01 & 0.64 & 172.85 & 9.14 & 11.60 & 4.92 & 4.51 & 1.87 & 0.32 & 0.54 \\
\hline Lump4 & 23.32 & 2579.66 & 73.21 & 9.55 & 53.18 & 16.62 & 31.14 & 1.14 & 1.63 & 3.68 & 1.65 & 0.65 \\
\hline Lump5 & 0.18 & 44.37 & 39.06 & 2845.00 & 0.08 & 0.92 & 0.83 & 0.96 & 0.91 & 0.00 & 151.00 & 123.65 \\
\hline
\end{tabular}




\section{Supplemental Table 8}

\section{Chemical Parameters of the 15 Lumps}

\section{Using k-means Clustering to Group the non-Target Chemicals}

\begin{tabular}{|c|c|c|c|c|c|c|c|c|c|c|c|c|}
\hline Name & Percent & ppmv & MW & PBA & PFat & PBrn & PLiv & PMsl & PKid & PLng & VmaxCL & $\mathrm{KmL}$ \\
\hline Lump1 & 18.83 & 2088.90 & 96.57 & 23.81 & 42.16 & 2.53 & 3.47 & 1.38 & 1.15 & 0.60 & 3.77 & 1.00 \\
\hline Lump2 & 0.24 & 36.98 & 43.09 & 0.02 & 1.00 & 51.74 & 106.14 & 1.00 & 1.00 & 11.45 & 0.19 & 0.10 \\
\hline Lump3 & 0.53 & 29.86 & 114.23 & 2.74 & 201.79 & 6.83 & 6.34 & 7.34 & 5.13 & 1.47 & 0.70 & 1.40 \\
\hline Lump4 & 1.36 & 101.74 & 83.96 & 0.61 & 190.35 & 8.02 & 8.97 & 6.42 & 4.86 & 1.74 & 0.36 & 0.64 \\
\hline Lump5 & 0.07 & 3.95 & 112.21 & 3.22 & 202.09 & 6.80 & 6.27 & 7.37 & 5.13 & 1.47 & 0.56 & 1.35 \\
\hline Lump6 & 3.79 & 424.32 & 56.51 & 0.07 & 79.74 & 19.46 & 34.40 & 1.00 & 2.32 & 4.28 & 0.20 & 0.18 \\
\hline Lump7 & 1.93 & 108.07 & 114.23 & 1.77 & 165.54 & 6.75 & 6.05 & 1.86 & 4.88 & 1.46 & 0.03 & 0.82 \\
\hline Lump8 & 42.68 & 3826.94 & 70.13 & 0.23 & 164.85 & 10.65 & 14.83 & 4.37 & 4.28 & 2.02 & 0.31 & 0.33 \\
\hline Lump9 & 3.00 & 224.36 & 86.18 & 0.35 & 245.53 & 10.50 & 12.43 & 6.34 & 5.60 & 2.53 & 0.20 & 0.47 \\
\hline Lump10 & 0.18 & 44.37 & 39.06 & 2845.00 & 0.08 & 0.92 & 0.83 & 0.96 & 0.91 & 0.00 & 151.00 & 123.65 \\
\hline Lump11 & 0.07 & 3.26 & 119.86 & 13.45 & 204.33 & 6.57 & 5.75 & 7.55 & 5.18 & 1.42 & 7.74 & 1.19 \\
\hline Lump12 & 5.10 & 346.91 & 98.99 & 1.32 & 197.64 & 7.26 & 7.29 & 7.01 & 5.03 & 1.57 & 0.43 & 1.03 \\
\hline Lump13 & 0.82 & 52.63 & 95.53 & 2.87 & 107.25 & 7.44 & 10.81 & 1.04 & 2.94 & 1.61 & 0.64 & 1.26 \\
\hline Lump14 & 1.92 & 135.42 & 92.18 & 1.08 & 208.86 & 8.17 & 8.61 & 7.06 & 5.54 & 1.50 & 0.45 & 0.79 \\
\hline Lump15 & 0.20 & 9.89 & 129.97 & 6.80 & 203.24 & 6.65 & 5.95 & 7.47 & 5.16 & 1.43 & 0.70 & 1.87 \\
\hline
\end{tabular}




\section{Supplemental Figure 1}
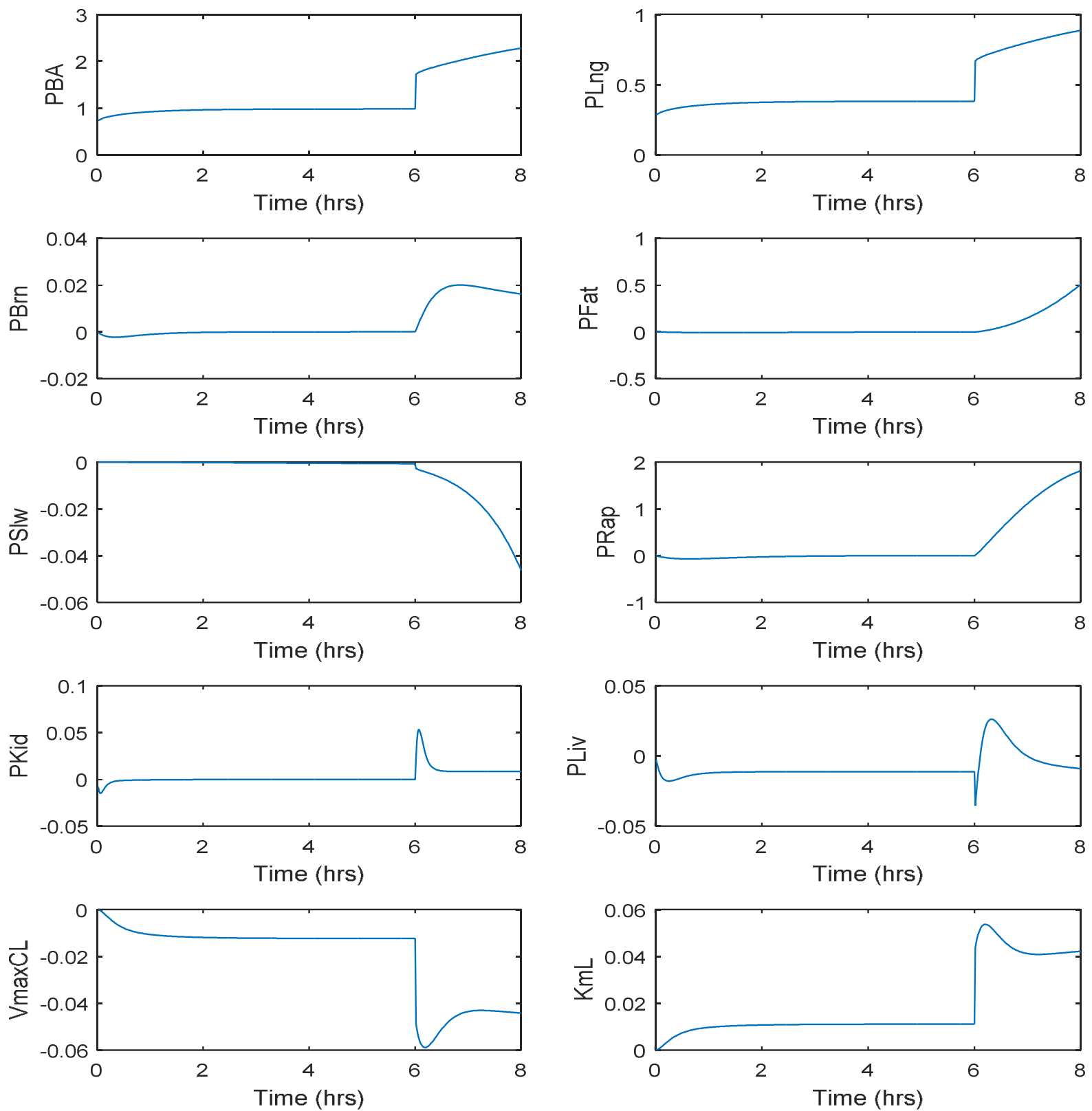

Supplemental Figure 1. Plots of the relative sensitivity of the amount of chemical in the arterial blood, AArt, to changes in the tissue partition coefficients and metabolism parameters Vmax and $\mathrm{Km}$, for the chemical Benzene. All parameters were set to 1.0 before running the analysis to obtain a normalized sensitivity. The plots show that the amount of chemical in the arterial blood, AArt, is most sensitive to the blood:air partition coefficient PBA, then the rapidly perfused tissues partition coefficient PRap, then the lung partition coefficient PLng, then the fat PFat, the kidney PKid, the liver PLiv, followed by Vmax and Km.

To run the sensitivity analysis, we used the Matlab code of Martin Fink and Adam Attarian which can be found in these references ${ }^{9,10}$. 


\section{Supplemental Figure 2}

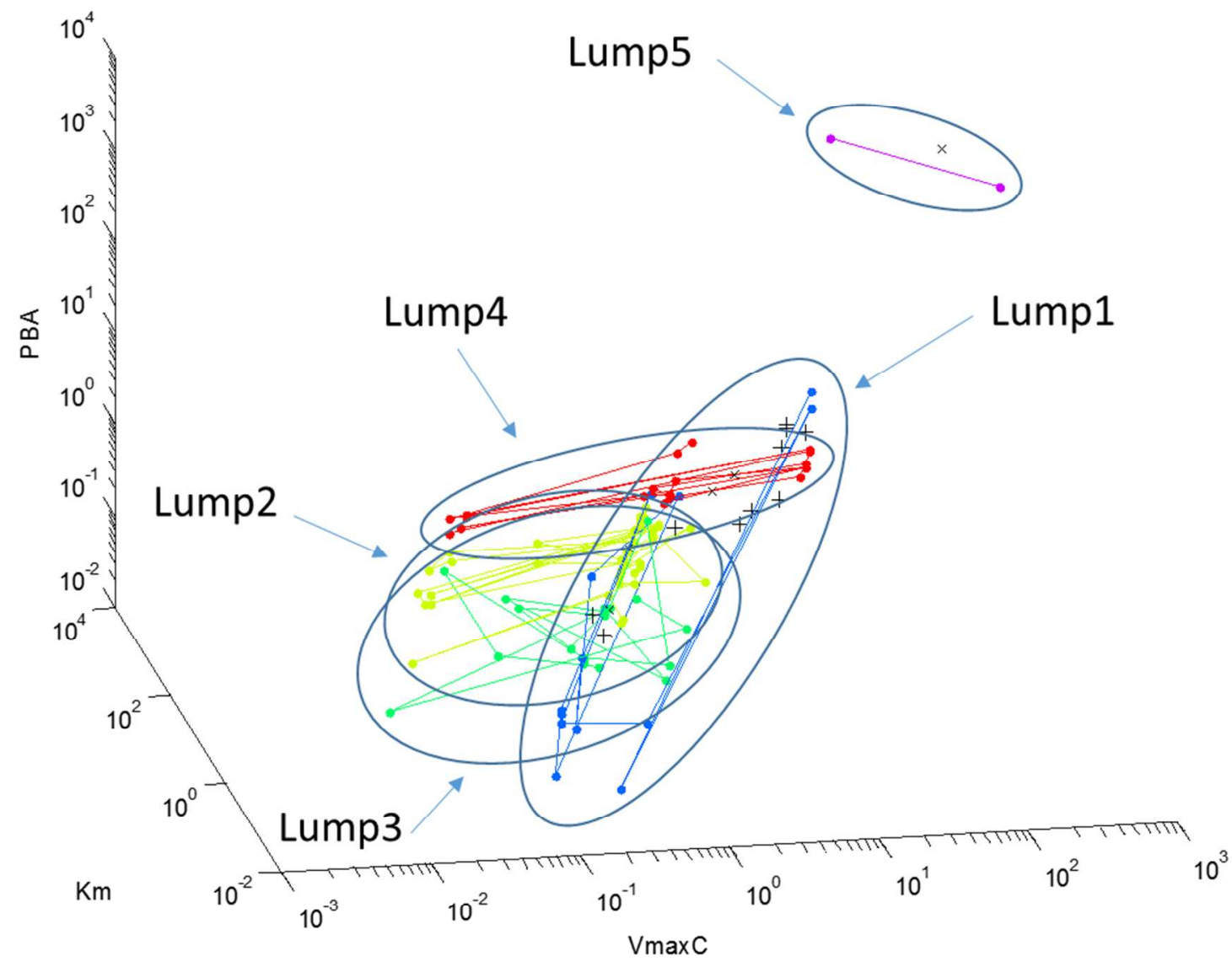

Supplemental Figure 2. Plot of 5 lumps clustered from all 109 identified and quantified chemicals. The k-means algorithm is used to cluster based on all chemical specific parameters, however, only three parameters are plotted for illustration. Target chemicals are plotted with vertical crosses + , non-target chemicals with dots $\cdot$, the center of each lump with diagonal crosses $\times$. The non-target chemicals that constitute the same lump are connected with a line. Each lump is plotted with a different color, circled, and labeled. The axes are the blood/air partition coefficient (PBA), the Michaelis-Menten metabolism term Km, and the VmaxC (Vmax is later allometrically scaled to body weight in the PBPK model, however, the unscaled $\mathrm{VmaxC}$ is shown in the figure). 


\section{Supplemental Figure 3a}
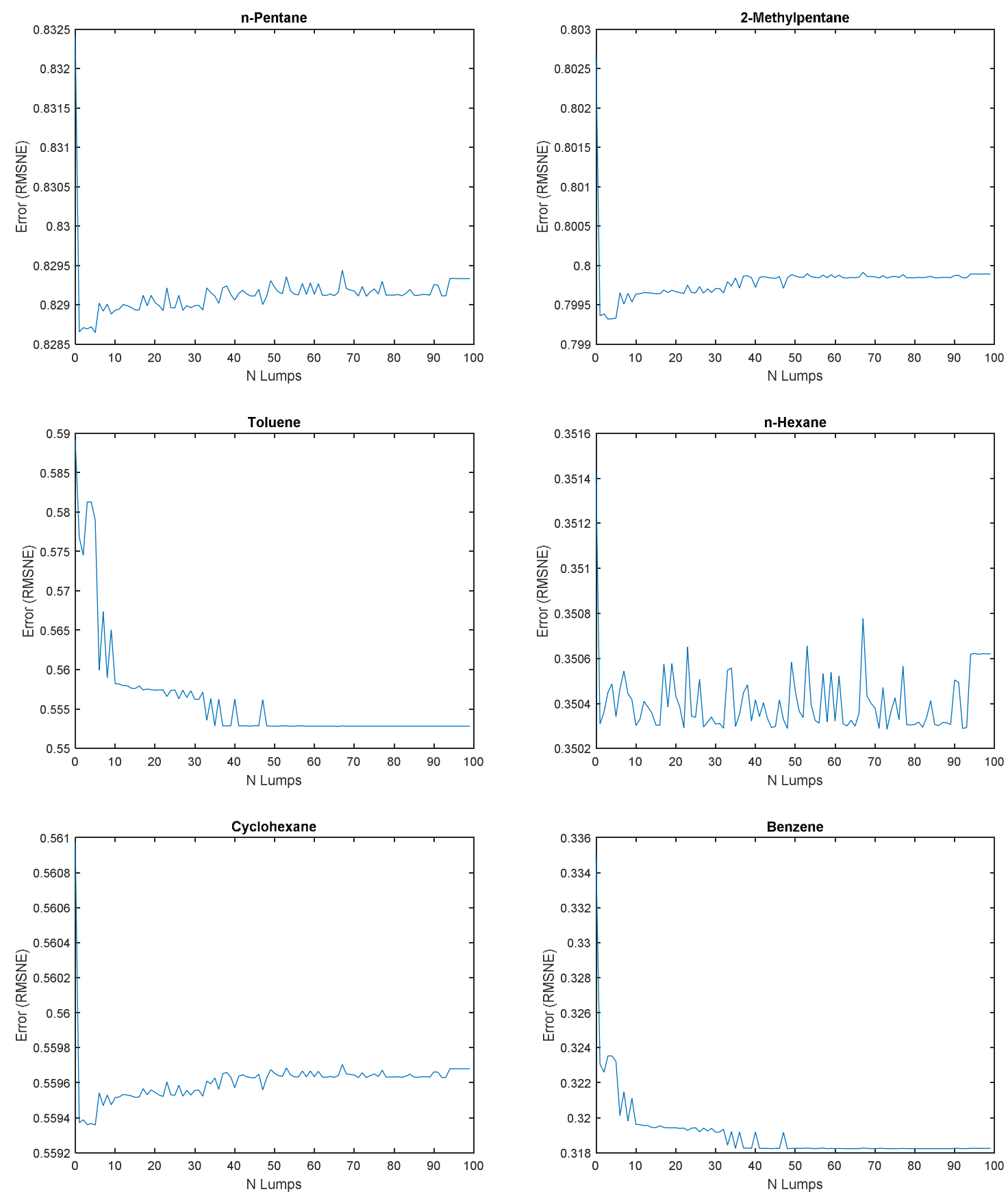


\section{Supplemental Figure 3b}
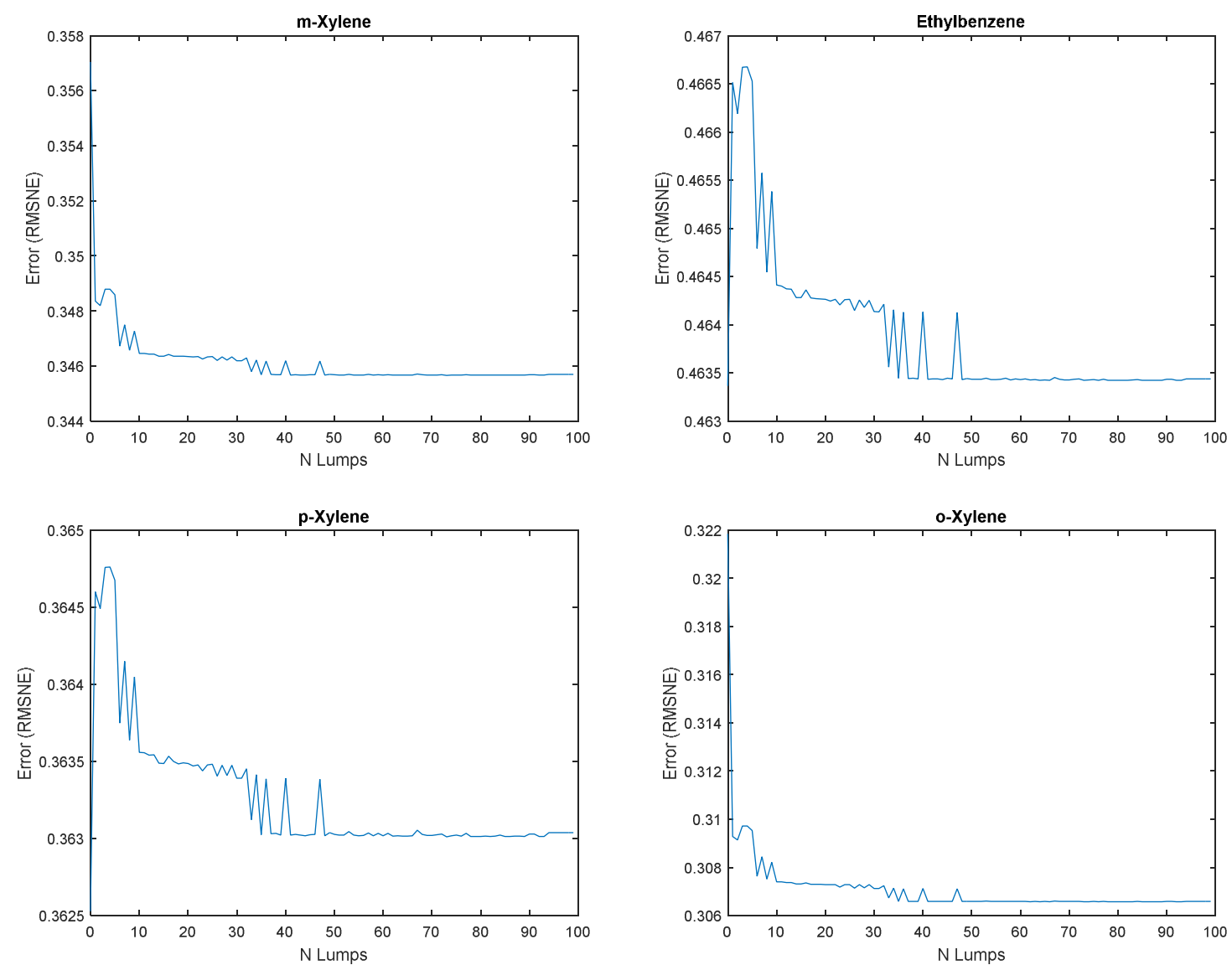

Supplemental Figure 3. Plots of simulation error, when simulating all 10 target chemicals interacting with each number of lumps $(\mathrm{N})$ from 0 to 99 . The error is calculated using the RMSNE equation comparing the simulated and experimental arterial blood concentrations for the 10 target chemicals: a) n-Pentane, 2-Methylpentane, Toluene, n-Hexane, Cyclohexane, Benzene, b) m-Xylene, Ethylbenzene, p-Xylene, and o-Xylene.

The discrete noise in the error plots is due to the k-mean clustering algorithm, because the lumping configuration differs slightly for each number of lumps $\mathrm{N}$.

For some slowly metabolized chemicals (e.g. n-Pentane, 2-Methylpentane, n-Hexane, Cyclohexane) the error increases with more lumps. However, the error is already so small that the relative change is small. 


\section{Supplemental Figure 4a}
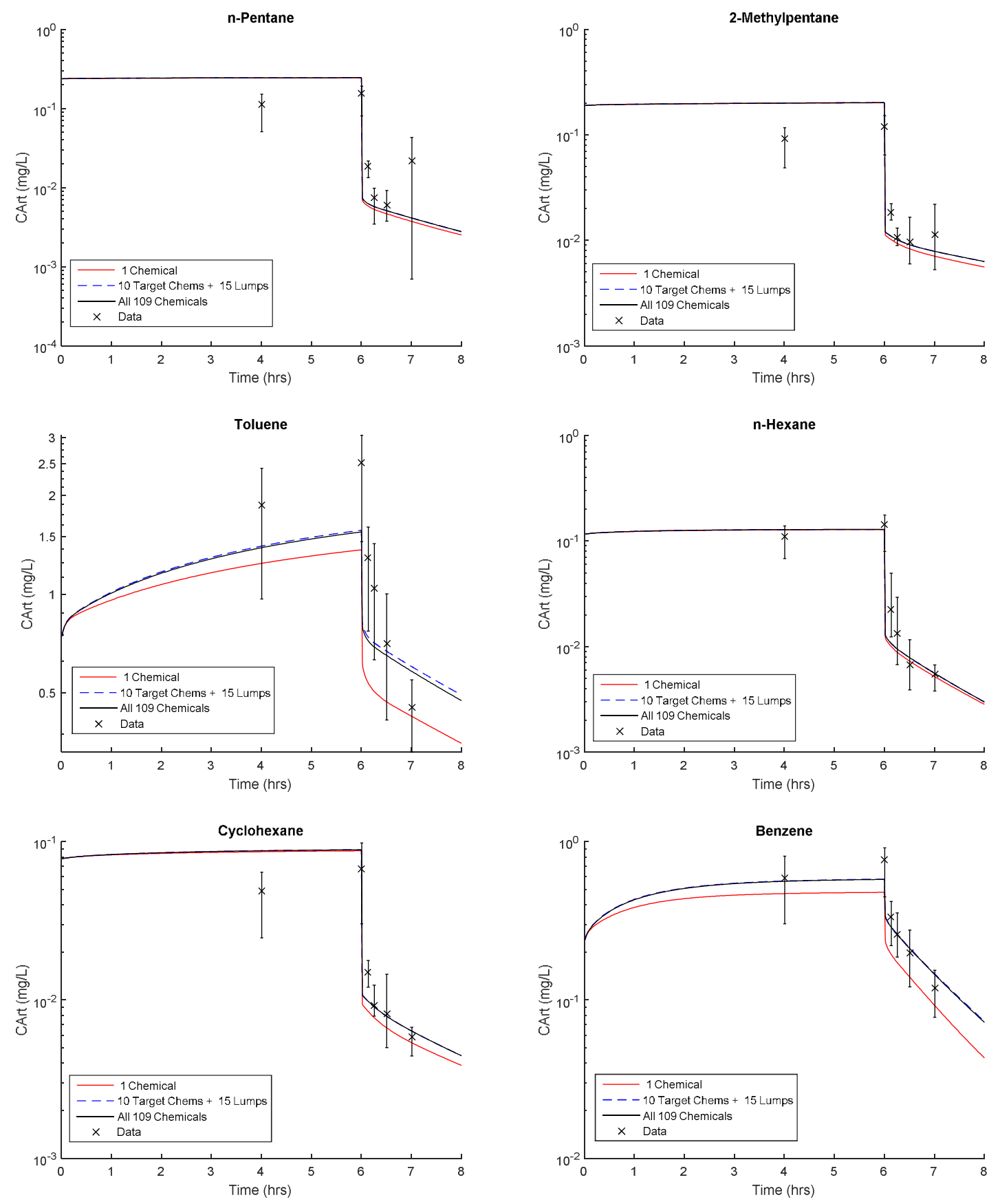


\section{Supplemental Figure 4b}
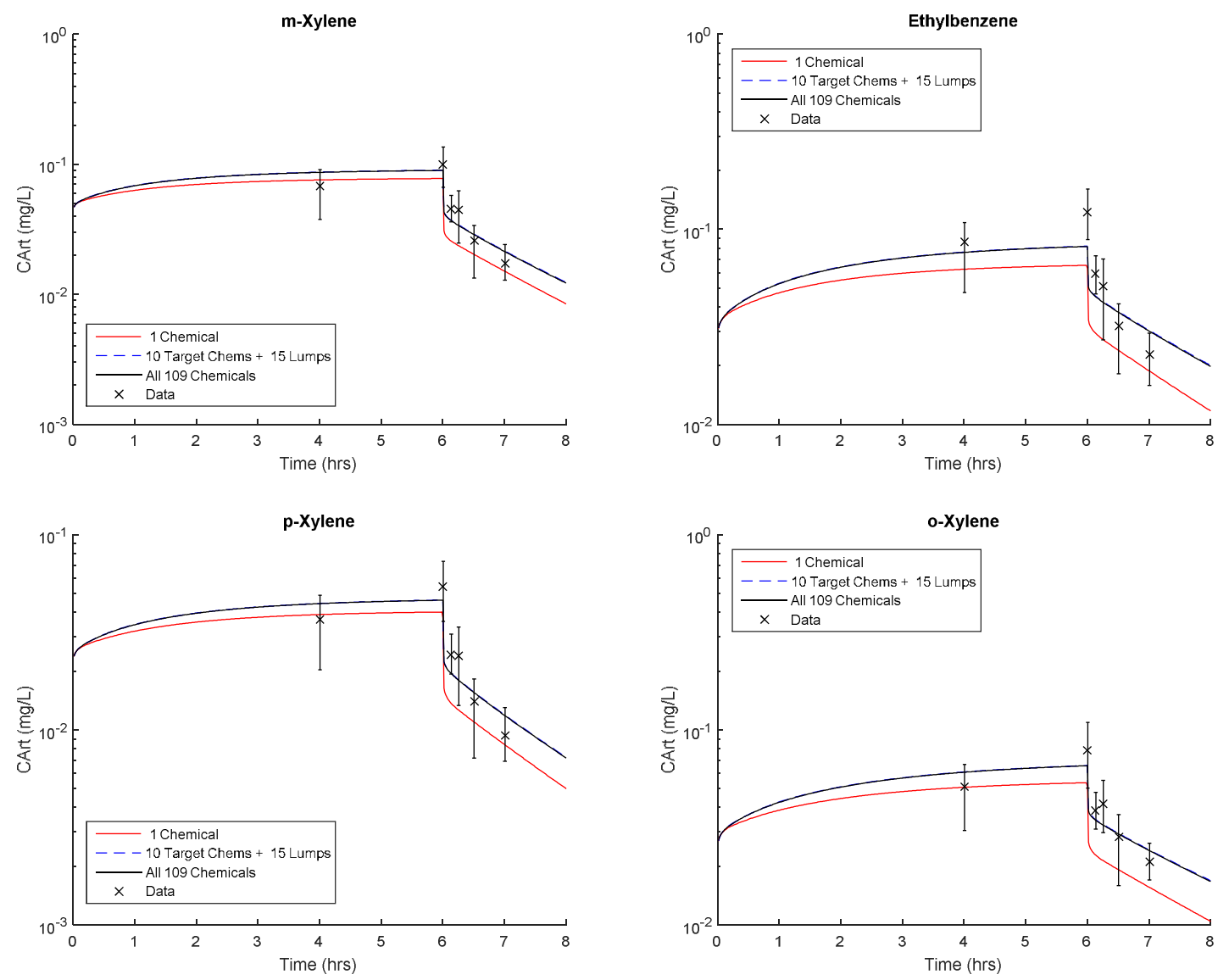

Supplemental Figure 4. Plots of the experimental data (crosses $\times$ ) and the simulated values (lines) for the arterial blood concentrations for each the 10 target chemicals when simulating 1) the single target chemical individually with no interaction, 2) 10 target chemicals and 15 lumps interacting (dashed line), and 3) all 109 known, measurable chemicals interacting. a) n-Pentane, 2-Methylpentane, Toluene, n-Hexane, Cyclohexane, Benzene, b) m-Xylene, Ethylbenzene, pXylene, and o-Xylene. The error bars show the maximum and minimum values of the experimental data. 


\section{Supplemental Figure 5}

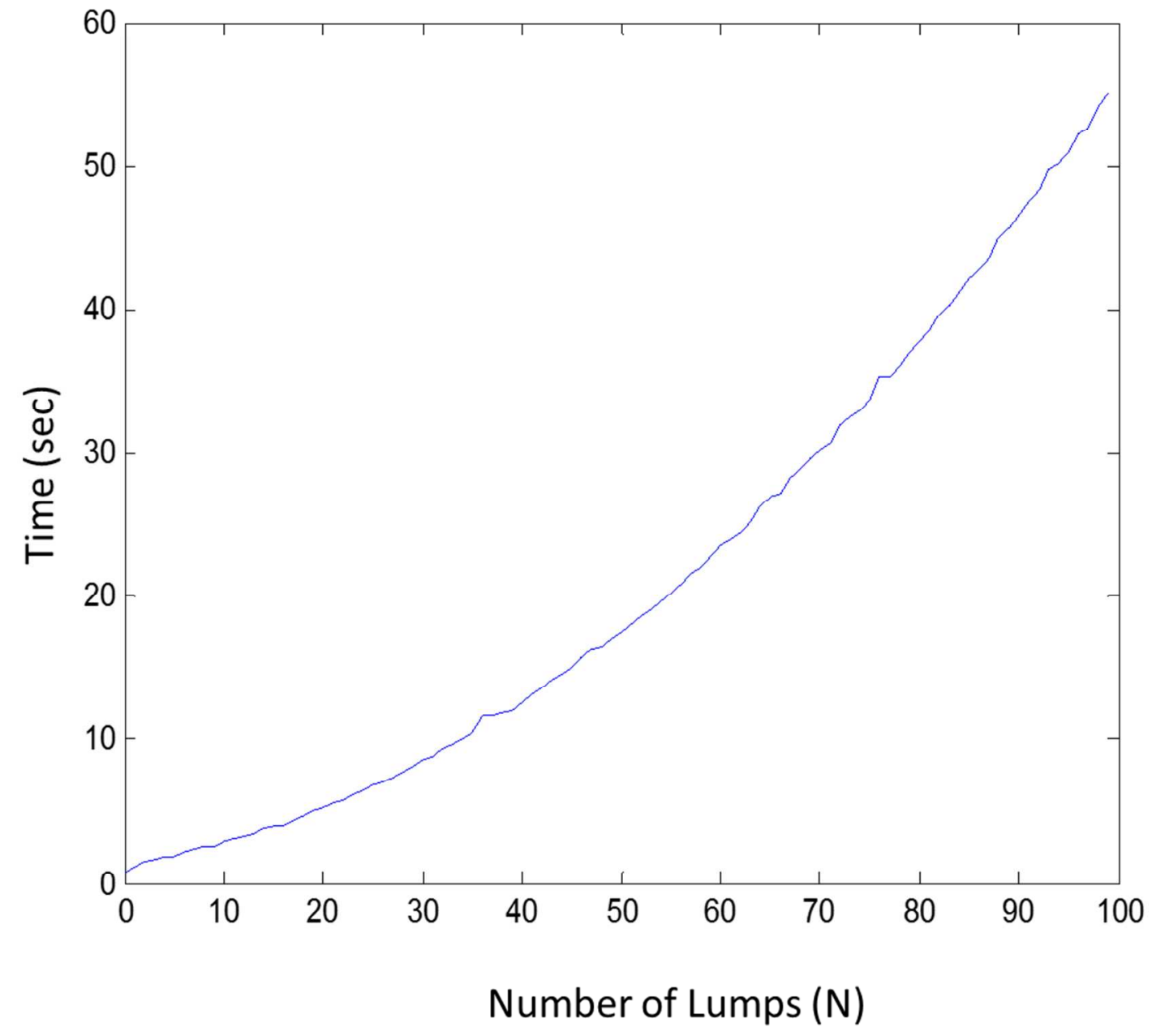

Supplemental Figure 5. Plot of the computational time when simulating all 10 target chemicals interacting with each number of lumps (N) from 0 to 99. 


\section{PBPK Model Equations}

These equations are calculated for each chemical i. Subscript $\mathrm{j}$ represents all other inhibiting chemicals.

$$
\begin{aligned}
& \mathrm{Km}_{\mathrm{i}}^{*}=\mathrm{Km}_{\mathrm{i}}\left(1+\sum_{\mathrm{j}} \frac{\mathrm{CLiv}_{\mathrm{j}}}{\mathrm{Km}_{\mathrm{j}}}\right) \\
& \operatorname{CVen}_{\mathrm{i}}=\left(\mathrm{QBrn}\left(\frac{\mathrm{CBrn}_{\mathrm{i}}}{\operatorname{PBrn}_{\mathrm{i}}}\right)+\mathrm{QFat}\left(\frac{\mathrm{CFat}_{\mathrm{i}}}{\mathrm{PFat}_{\mathrm{i}}}\right)+\mathrm{QSlw}\left(\frac{\mathrm{CSlw}_{\mathrm{i}}}{\operatorname{PSlw}_{\mathrm{i}}}\right)+\mathrm{QRap}\left(\frac{\mathrm{CRap}_{\mathrm{i}}}{\operatorname{PRap}_{\mathrm{i}}}\right)+\mathrm{QKid}\left(\frac{\mathrm{CKid}_{\mathrm{i}}}{\operatorname{PKid}_{\mathrm{i}}}\right)+\mathrm{QLiv}\left(\frac{\operatorname{CLiv}_{\mathrm{i}}}{\operatorname{PLiv}_{\mathrm{i}}}\right)\right) / \mathrm{QTot} \\
& \text { RAArt }_{i}=\operatorname{QPul}\left(\operatorname{CInh}_{\mathrm{i}}-\frac{\mathrm{CArt}_{\mathrm{i}}}{\mathrm{PBA}_{\mathrm{i}}}\right)+\operatorname{QTot}\left(\mathrm{CVen}_{\mathrm{i}}-\frac{\mathrm{CArt}_{\mathrm{i}}}{\operatorname{PLng}_{\mathrm{i}}}\right) \\
& \operatorname{RABrn}_{\mathrm{i}}=\operatorname{QBrn}\left(\mathrm{CArt}_{\mathrm{i}}-\frac{\mathrm{CBrn}_{\mathrm{i}}}{\operatorname{PBrn}_{\mathrm{i}}}\right) \\
& \text { RAFat }_{i}=\text { QFat }\left(\text { CArt }_{\mathrm{i}}-\frac{\text { CFat }_{\mathrm{i}}}{\text { PFat }_{\mathrm{i}}}\right) \\
& \operatorname{RASlw}_{\mathrm{i}}=\operatorname{QSlw}\left(\operatorname{CArt}_{\mathrm{i}}-\frac{\operatorname{CSlw}_{\mathrm{i}}}{\operatorname{PSlw}_{\mathrm{i}}}\right) \\
& \operatorname{RARap}_{\mathrm{i}}=\operatorname{QRap}\left(\operatorname{CArt}_{\mathrm{i}}-\frac{\mathrm{CRap}_{\mathrm{i}}}{\operatorname{PRap}_{\mathrm{i}}}\right) \\
& \operatorname{RAKid}_{\mathrm{i}}=\operatorname{QKid}\left(\operatorname{CArt}_{\mathrm{i}}-\frac{\mathrm{CKid}_{\mathrm{i}}}{\operatorname{PKid}_{\mathrm{i}}}\right) \\
& \operatorname{RAMLiv}_{\mathrm{i}}=\frac{\operatorname{Vmax}_{\mathrm{i}} \cdot \operatorname{CLiv}_{\mathrm{i}}}{\operatorname{Km}_{\mathrm{i}}^{*}+\operatorname{CLiv}_{\mathrm{i}}} \\
& \operatorname{RALiv}_{\mathrm{i}}=\operatorname{QLiv}\left(\operatorname{CArt}_{\mathrm{i}}-\frac{\mathrm{CLiv}_{\mathrm{i}}}{\operatorname{PLiv}_{\mathrm{i}}}\right)-\operatorname{RAMLiv}_{\mathrm{i}}
\end{aligned}
$$

Where $\mathrm{R}$ indicates a rate (dA/dt), A indicates an amount, $\mathrm{C}$ a concentration, $\mathrm{V}$ a volume, $\mathrm{Q}$ a flowrate, $\mathrm{P}$ a partition coefficient, Ven is the venous blood, Art the arterial blood, Brn the brain, Fat the fat/adipose Tissue, Slw the slowly perfused tissues, Rap the rapidly perfused tissues. Kid the kidney, Liv the liver, AM the amount metabolized, PBA the blood:air partition coefficient, QPul the pulmonary ventilation rate, QTot the total blood circulation rate, and CInh the concentration of chemical inhaled.

The concentrations are calculated as the amount divided by volume, for example:

$\mathrm{CBrn}_{\mathrm{i}}=\frac{\mathrm{ABrn}_{\mathrm{i}}}{\mathrm{VBrn}}$ 


\section{References}

1. Meulenberg, C. J.; Vijverberg, H. P., Empirical relations predicting human and rat tissue:air partition coefficients of volatile organic compounds. Toxicology and applied pharmacology 2000, 165, (3), 206-16.

2. Price, K.; Krishnan, K., An integrated QSAR-PBPK modelling approach for predicting the inhalation toxicokinetics of mixtures of volatile organic chemicals in the rat. SAR and QSAR in environmental research 2011, 22, (1-2), 107-28.

3. Perbellini, L.; Brugnone, F.; Caretta, D.; Maranelli, G., Partition coefficients of some industrial aliphatic hydrocarbons (C5-C7) in blood and human tissues. British journal of industrial medicine 1985, 42, (3), 162-7.

4. Beliveau, M.; Tardif, R.; Krishnan, K., Quantitative structure-property relationships for physiologically based pharmacokinetic modeling of volatile organic chemicals in rats. Toxicology and applied pharmacology 2003, 189, (3), 221-32.

5. Pence, H. E.; Williams, A., ChemSpider: An Online Chemical Information Resource. J Chem Educ 2010, 87, (11), 1123-1124.

6. USEPA EPISuite. http://www.epa.gov/opptintr/exposure/pubs/episuite.htm (June 2015),

7. Abraham, M. H.; Ibrahim, A.; Acree, W. E., Jr., Air to blood distribution of volatile organic compounds: a linear free energy analysis. Chemical research in toxicology 2005, 18, (5), 904-11.

8. Paterson, S.; Mackay, D., Correlation of tissue, blood, and air partition coefficients of volatile organic chemicals. British journal of industrial medicine 1989, 46, (5), 321-8.

9. Attarian, A. Using tssolve.m for Sensitivity Analysis and Least Squares Estimation. http://www4.ncsu.edu/ arattari/tssolveman.pdf

10. Fink, M. Automatic Differentiation for Matlab. http://www.mathworks.com/matlabcentral/fileexchange/15235-automatic-differentiation-for$\underline{\text { matlab }}$ 